\title{
Probing the cosmological variation of the fine-structure constant: Results based on VLT-UVES sample ${ }^{\star}$
}

\author{
H. Chand ${ }^{1}$, R. Srianand ${ }^{1}$, P. Petitjean ${ }^{2,3}$, and B. Aracil ${ }^{2}$ \\ ${ }^{1}$ IUCAA, Post Bag 4, Ganeshkhind, Pune 411007, India \\ 2 Institut d'Astrophysique de Paris - CNRS, 98bis Boulevard Arago, 75014 Paris, France \\ 3 LERMA, Observatoire de Paris, 61 Rue de l'Observatoire, 75014 Paris, France
}

Received 17 November 2003 / Accepted 5 January 2004

\begin{abstract}
Development of fundamental physics relies on the constancy of various fundamental quantities such as the finestructure constant. Detecting or constraining the possible time variations of these fundamental physical quantities is an important step toward a complete understanding of basic physics. High-quality absorption lines seen in the spectra of distant QSOs allow one to probe time variations of several of these quantities. Here we present the results from a detailed manymultiplet analysis, to detect the possible variation of the fine-structure constant, performed using high signal-to-noise ratio, ( $~ 70$ per pixel), high spectral resolution $(R \geq 45000)$ observations of $23 \mathrm{Mg}$ II systems detected toward 18 QSOs in the redshift range $0.4 \leq z \leq 2.3$ obtained using UVES at the VLT. We validate our procedure and define the selection criteria that will avoid possible systematics using a detailed analysis of a simulated data set. The spectra of Mg II doublets and Fe II multiplets are generated considering variations in $\alpha$ and specifications identical to that of our UVES spectra. We show that our Voigt profile fitting code recovers the variation in $\alpha$ very accurately when we use single component systems and multiple-component systems that are not heavily blended. Spurious detections are frequently seen when we use heavily blended systems or systems with very weak lines. Thus we avoided these system while analysing the UVES data. To make the analysis transparent and accessible to the community for critical scrutiny, all the steps involved in the analysis are presented in detail. The weighted mean value of the variation in $\alpha$ obtained from our analysis over the redshift range $0.4 \leq z \leq 2.3$ is $\Delta \alpha / \alpha=(-0.06 \pm 0.06) \times 10^{-5}$. The median redshift of our sample is 1.55 and corresponds to a look-back time of $9.7 \mathrm{Gyr}$ in the most favored cosmological model today. The $3 \sigma$ upper limit on the time variation of $\alpha$ is $-2.5 \times 10^{-16} \mathrm{yr}^{-1} \leq(\Delta \alpha / \alpha \Delta t) \leq+1.2 \times 10^{-16} \mathrm{yr}^{-1}$. To our knowledge this is the strongest constraint from quasar absorption line studies to till date.
\end{abstract}

Key words. galaxies: quasars: absorption lines - cosmology: observations

\section{Introduction}

Contemporary theories of fundamental interactions, such as SUSY GUT or Super-string theories, treating gravity and quantum mechanics in a consistent way, not only predict a dependence of fundamental physical constants on energy (which has been observed in high energy experiments) but also allow their cosmological time and space variations (Uzan 2003). Detecting or constraining the possible time variations of fundamental physical quantities is an important step toward a complete understanding of basic physics. In the framework of standard Big-bang models one can probe the evolution of various physical quantities over the elapse time using measurements that are performed at different redshifts $(z)$. As the

Send offprint requests to: $\mathrm{R}$. Srianand,

e-mail: anand@iucaa.ernet.in

* Based on observations collected at the European Southern Observatory (ESO), under the Large Programme "QSO Absorption Line Systems" ID No. 166.A-0106 with UVES on the 8.2 m KUEYEN telescope operated at the Paranal Observatory, Chile. energy of atomic transitions depends on the electromagnetic coupling constant $\alpha$ (its well measured laboratory value is $e^{2} / \hbar c=1 / 137.03599976(50)$, see Mohr \& Taylor 2000), its possible time variation will be registered in the form of small shifts in the absorption line spectra seen toward high-z QSOs (Bahcall et al. 1967). Initial attempts to measure the variation in $\alpha$ were based on alkalidoublets (Wolfe et al. 1976; Levshakov 1994; Potekhin \& Varshalovich 1994; Cowie \& Songaila 1995; Murphy et al. 2001). This method (AD method) uses the difference in the wavelengths of the doublets originating from the same ground state (i.e., ${ }^{2} \mathrm{~S}_{1 / 2} \rightarrow{ }^{2} \mathrm{P}_{3 / 2}$ and ${ }^{2} \mathrm{~S}_{1 / 2} \rightarrow{ }^{2} \mathrm{P}_{1 / 2}$ transitions). The constraints on the variation in $\alpha, \Delta \alpha / \alpha$, are obtained by assuming that the measured difference in the wavelength centroid of the doublets is proportional to $\alpha^{2}$ to the lowest order. The best constraint obtained using this method is $\Delta \alpha / \alpha=(-0.5 \pm 1.3) \times 10^{-5}$ (Murphy et al. 2001). Like absorption doublets one can also use the central wavelength of multiple emission lines originating from the same initial excited state. For example, Bahcall et al. (2004) use the nebular 
$\mathrm{O}$ III emission lines at $\lambda 5007$ and $\lambda 4959$ originating from ${ }^{1} \mathrm{D}_{2}$ excited level and derive $\Delta \alpha / \alpha=(0.7 \pm 1.4) \times 10^{-4}$ based on 73 quasar spectra over the redshift range $0.16 \leq z \leq 0.80$. Studies based on molecular absorption lines seen in the radio/mm wavelength range are more sensitive than those based on optical/UV absorption lines. At present good constraints are available for two systems, $\Delta \alpha / \alpha=(-0.10 \pm 0.22) \times 10^{-5}$ at $z=$ 0.2467 and $\Delta \alpha / \alpha=(-0.08 \pm 0.27) \times 10^{-5}$ at $z=0.6847$ if one assumes constant proton $g$-factor (Murphy et al. 2001a). It is also possible to have joint constraints on the fundamental constants using molecular lines (see Chengalur \& Kanekar 2003). However, such studies at high $z$ are not available due to the lack of molecular absorption systems at high $z$. The most suitable and accurate method for measuring $\Delta \alpha / \alpha$ at high redshift is called the many-multiplet method (MM method). This is a generalization of the AD method and was introduced by Dzuba et al. (1999). Unlike the AD method, this method uses absolute wavelength measurements of numerous absorption lines from different species. This method has been shown to provide an order of magnitude improvement in the measurement of $\Delta \alpha / \alpha$ compared to AD method (Murphy et al. 2003, and references therein). However, this method is also extremely vulnerable to a number of systematical errors. Accurate laboratory wavelengths (up to an accuracy of few $\mathrm{m} \AA$ ) are available for most of the important transitions that are regularly detected in QSO spectra (see Table 1). The sensitivity coefficients of the different line transitions from different multiplets to the variation in $\alpha$ were computed using many-body calculations taking into account the dominant relativistic effects (Dzuba et al. 1999, 2002). If one uses the parameterization of these authors then the accuracy in probing the possible variations in $\alpha$ depends very much on how well one measures the wavelength of the absorption lines at high $z$. In simple terms, the MM method exploits the fact that the energy of different line transitions varies differently for a given change in $\alpha$. For example, rest wavelengths of $\mathrm{Mg}$ II doublets and $\mathrm{Mg}$ I are fairly insensitive to small changes in $\alpha$, thereby providing good anchors for measuring the systemic redshift. On the other hand, the rest wavelengths of Fe II multiplets are very sensitive to small variations in $\alpha$. Thus constraining relative shifts between an anchor and different Fe II lines make it possible to study the variations in $\alpha$. However the accuracy depends on how well the absorption line profiles are modeled. The absorption profiles are usually modeled using multiple Voigt profiles that are defined by column density $(N)$, velocity dispersion $(b)$ and redshift in addition to the rest-wavelength of the species. In general the Voigt profile decomposition is robust. However, the profile decomposition is not unique when the absorption lines are heavily blended and/or if the signal-to-noise ratio of the spectrum is not high enough. In addition, in real data, small relative shifts between absorption lines from different species can be introduced due to various systematic effects such as chemical and ionization inhomogeneities in the absorbing region, incorrect wavelength calibration, isotopic abundances, and atmospheric dispersion effects etc. It is usually argued that these random systematic effects can be canceled by using a large number of measurements. Indeed, applying the method to 128 systems over a large redshift range $0.2<z<3.7$, Murphy et al. (2003) measured
$\Delta \alpha / \alpha=(-0.54 \pm 0.12) \times 10^{-5}$. The detailed work based on the MM method has been performed by only one group using simultaneous fitting of absorption line profiles of species covering a wide range of ionization states. Their important results need to be confirmed independently using a data set that is optimized for the purpose. This forms the main motivation of this work. One of the main criticism made of the MM method is the apparent lack of transparency in the complex analysis (Bahcall et al. 2004). Here, we perform a similar analysis to the one used by Webb and collaborators on a homogeneous and very high quality data set. To make the whole process accessible for critical scrutiny, we give a detail description of the procedure and the individual fits. The main idea is that it is better to derive strong constraints from small, though statistically significant, sample of well chosen systems rather than loose constraints from a larger sample of strongly blended systems. The details of the observations and the quality of the data used in the analysis are discussed in Sect. 2. In Sect. 3 we discuss simulations that are performed to validate our Voigt profile fitting code in extracting the variations in $\alpha$. We also use the simulation results to construct an optimum selection criterion that will ensure the best possible detection limit in $\Delta \alpha / \alpha$. In Sect. 4 we list all the $\mathrm{Mg}$ II, Fe II systems that are selected in our sample. Comments on individual Voigt profile fitting are given in Sect. 5. The discussion and conclusions are presented in Sect. 6.

\section{Observations and data sample}

The data used in this study have been obtained using the Ultraviolet and Visible Echelle Spectrograph (UVES) mounted on the ESO KUEYEN $8.2 \mathrm{~m}$ telescope at the Paranal observatory for the ESO-VLT Large Programme "QSO absorption lines". This corresponds to a homogeneous sample of 18 QSO lines of sight suitable for studying various properties of the intergalactic medium over a redshift range 1.7-3.2. All the objects were observed in good seeing conditions with 1 arcsec slit width. The data were reduced using the UVES pipeline, a set of procedures implemented in a dedicated context of MIDAS, the ESO data reduction package. The main function of the pipeline is to perform a precise inter-order background subtraction for science frames and master flat-fields, and to allow an optimal extraction of the object signal, rejecting cosmic ray impacts and performing sky-subtraction at the same time. Usually a given wavelength range is covered by more than 6 exposures, and removal of cosmic rays and odd flux fluctuations in a given exposure is robust. The reduction is checked step by step. Wavelengths are corrected to vacuum-heliocentric values and individual 1D spectra are combined. Air-vacuum conversions and heliocentric corrections were done using standard conversion equations (Elden 1966; Stumpff 1980). The slit was always oriented along the parallactic angle, and calibration exposures were taken before or after the scientific exposures. Thus most of the important concerns raised by Murphy et al. (2003), are taken care of in our observations. As we are using the $\chi^{2}$ minimization method to detect sub-pixel-scale shifts, the error spectrum is crucial for our analysis. Great care was taken in computing the error spectrum while combining the individual exposures. Our final error is the quadratic sum of appropriately 
Table 1. Summary of atomic parameters that are used in our analysis.

\begin{tabular}{|c|c|c|c|c|c|c|c|}
\hline Species & $\lambda_{0}(\AA)$ & Ref. $\left(\lambda_{0}\right)$ & $\overline{\omega_{0}\left(\mathrm{~cm}^{-1}\right)}$ & $q$ & id & $\bar{f}$ & Ref. $(f)$ \\
\hline $\operatorname{Mg} I$ & $2852.96310(8)$ & $\mathrm{L}$ & $35051.277(1)$ & $+86(10)$ & $\mathrm{i}$ & 1.830 & $\mathrm{~A}$ \\
\hline \multirow[t]{2}{*}{ Mg II } & $2796.3543(2)$ & $\mathrm{L}$ & $35760.848(2)$ & $+211(10)$ & $\mathrm{a}$ & 0.6123 & A \\
\hline & $2803.5315(2)$ & $\mathrm{L}$ & $35669.298(2)$ & $+120(10)$ & $\mathrm{b}$ & 0.3054 & A \\
\hline Al II & $1670.7887(1)$ & M & $59851.972(4)$ & $+270(30)$ & $\mathrm{j}$ & 1.88 & B \\
\hline \multirow[t]{2}{*}{ Si II } & $1526.70709(2)$ & M & $65500.4492(7)$ & $+68(30)$ & $\mathrm{k}$ & 0.133 & A \\
\hline & 1808.01301(1) & M & $55309.3365(4)$ & $+531(30)$ & 1 & 0.00208 & A \\
\hline \multirow[t]{6}{*}{$\mathrm{Fe}$ II } & $1608.45085(8)$ & $\mathrm{P}$ & $62171.625(3)$ & $-1200(300)$ & $\mathrm{c}$ & 0.0577 & $\mathrm{C}$ \\
\hline & $2344.2130(1)$ & Q & $42658.2404(2)$ & $+1254(150)$ & $\mathrm{d}$ & 0.114 & $\mathrm{D}$ \\
\hline & $2374.4603(1)$ & Q & $42114.8329(2)$ & $+1640(150)$ & $\mathrm{e}$ & 0.0313 & $\mathrm{D}$ \\
\hline & $2382.7642(1)$ & Q & $41968.0642(2)$ & $+1498(150)$ & $\mathrm{f}$ & 0.320 & $\mathrm{D}$ \\
\hline & $2586.6496(1)$ & Q & $38660.0494(2)$ & $+1520(150)$ & $\mathrm{g}$ & 0.0691 & $\mathrm{D}$ \\
\hline & $2600.1725(1)$ & Q & $38458.9871(2)$ & $+1356(150)$ & $\mathrm{h}$ & 0.239 & $\mathrm{D}$ \\
\hline
\end{tabular}

Reference:

oscillator strength:- (A) Morton (1991); (B) Prochaska et al. (2001); (C) Morton (2003); (D) Bergeson et al. (1996); wavelength:- (L) Pickering et al. (1998); (M) Griesmann \& Kling (2002); (P) Pickering et al. (2002); (Q) Nave et al. (1991).

interpolated weighted mean errors and possible errors due to scatter in the individual flux measurements. In all cases, the spectra cover the wavelength range of 3000-10000 ̊. A firstguess continuum is fitted with an automatic continuum fitting code. However, for the systems that are eventually used in the analysis, a local continuum normalization is performed using lower order polynomials. In all cases we have used the flux in the core of the saturated lines to estimate the background subtraction uncertainties. This corrections to the background subtraction uncertainties are applied whenever needed. We notice that continuum fitting is not unique in the Ly $\alpha$ forest region and in the IR region where atmospheric lines are plenty. In our analysis we have therefore avoided the absorption lines that lie on the lower wavelength side of Ly $\alpha$ emission from the quasar and lines that could be blended with atmospheric lines.

The properties of the spectra in our sample are summarized in Fig. 1. A typical $S / N \sim 60-80$ per pixel (typically $0.035 \AA$ wide) is achieved in the whole wavelength range of interest (see left-hand top panel in Fig. 1). This is a factor 2 improvement compared to the one used earlier for the analysis of $\Delta \alpha / \alpha$ (see Fig. 2 of Murphy et al. 2003). The spectral resolution measured from the lamp spectrum is typically $\geq 44000$.

We have investigated the accuracy of the wavelength calibration using lamp spectra that are extracted in the same way as the object. We notice that individual emission lines are well modeled by a Gaussian. This confirms that the instrumental profile can be modeled with a single Gaussian while fitting the Voigt profiles. The median difference between the extracted and the actual wavelengths at most of the settings are close to zero. The root mean square of this deviation is always smaller than $3 \mathrm{~m} \AA$ at all the settings. We confirm the robustness of the wavelength calibration using other consistency checks. In particular, the wavelengths of Fe II transitions strongly depend on $\alpha$ but the relative position of the lines (apart from Fe II $\lambda 1608$ ) does not depend much on $\alpha$. This means that, whatever the variation of $\alpha$ is, the relative velocity shifts between Fe II transitions should be consistent with zero in the case of good calibration. This is precisely what is observed (Panel $b$ in Fig. 1). Note in addition that the Fe II rest wavelengths are known to very high accuracy $(\sim 0.1 \mathrm{~mA})$. In principle a similar exercise can be performed using Si IV and C IV absorption lines. However, unlike Fe II lines, C IV wavelengths are known only to $2 \mathrm{~m} \AA$ accuracy. Even in this case the velocity shifts measured are consistent with zero within the allowed laboratory wavelength uncertainties (Petitjean \& Aracil 2003). When fitting the narrow doublets and multiplets from a given species in individual settings, we have allowed for some adjustments in the instrumental resolution. This seems to be an important step as we realized that the seeing was much better than the slit width during most of the observing run. The metal line systems in the reduced spectra are identified using the standard procedure.

The next step is to validate our Voigt profile fitting codes and make some estimate of various possible systematic effects. To do this we first simulate spectra with specifications similar to a typical UVES spectrum and perform the analysis on them. The details of the spectral simulations and the data analysis process is described in the following section.

\section{Chasing the systematics using simulated data}

As one tries to measure $\Delta \alpha / \alpha$ through Voigt profile decomposition of absorption lines, various systematic effects can cause false-alarm detection of $\Delta \alpha / \alpha$. The effects of random systematics can be averaged out by combining large numbers of measurements. However, the best strategy will be to have an a priori estimate of various systematic effects so that the proper selection criteria can be applied while choosing the sample to achieve the best possible results. In this section we try to understand various effects using simulated spectra. Such an exercise is also important to see how well our Voigt profile fitting code 

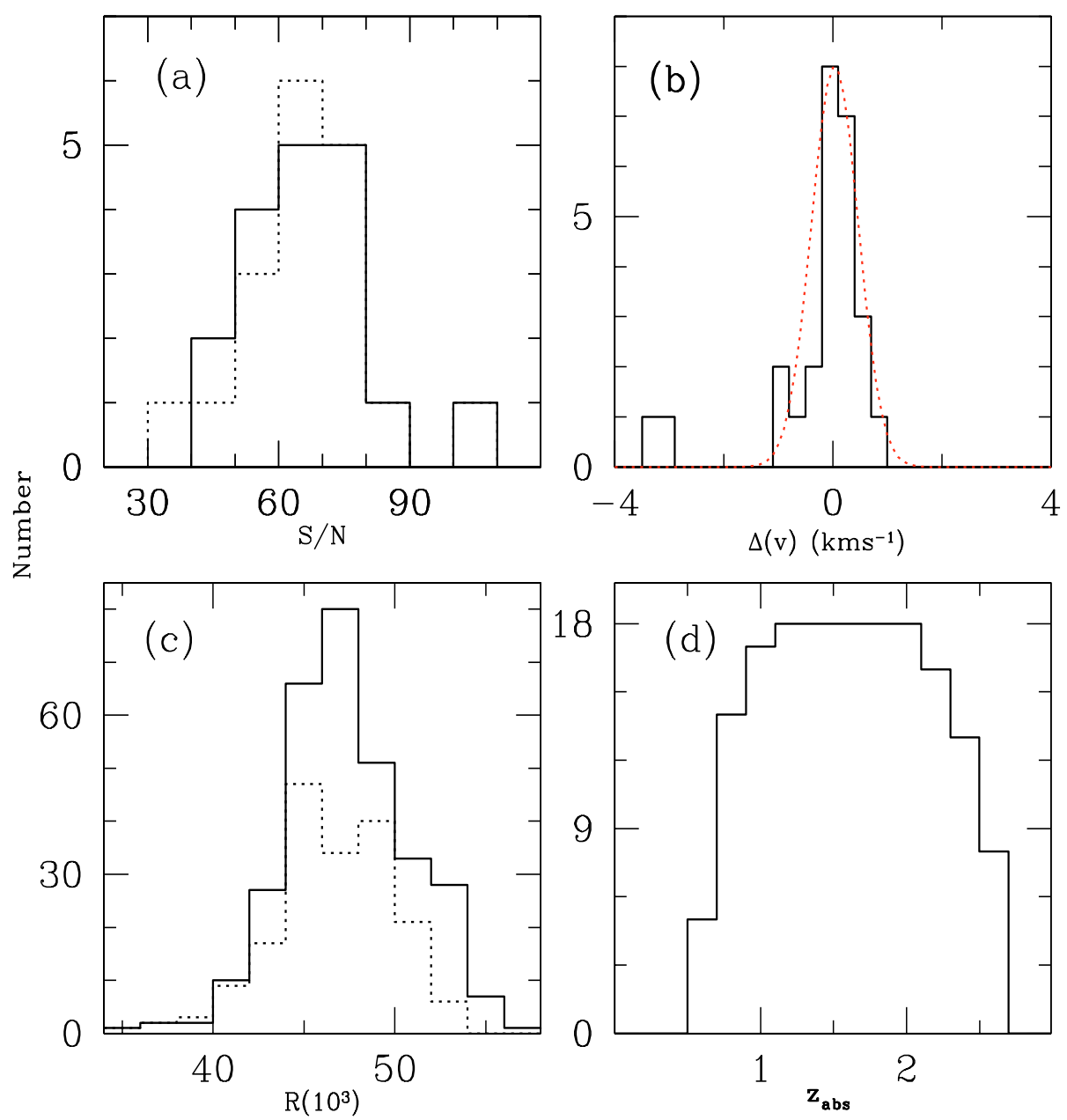

Fig. 1. Properties of our sample: Panel a) shows the distribution of median signal-to-noise $(S / N)$ ratio per pixel computed over $30 \AA$ intervals. A typical $S / N=60-80$ per pixel is achieved over the wavelength range 4000-8000 $\AA$. Panel b) shows the distribution of measured relative velocity shifts between the Fe II $\lambda 2344$ and Fe II $\lambda 2600$ lines in our sample. As the $q$ coefficients are similar for these transitions the velocity shift is expected to be distributed around zero in the case of good wavelength calibrations. Thus the plot provides an internal consistency check for the wavelength calibration. We notice that the accuracy in the wavelength calibration is of the order of $1-3 \mathrm{~m} \AA$ in the wavelength range of interest. Panel c) shows the distribution of the spectral resolution measured from the calibration spectrum. The typical spectral resolution achieved is

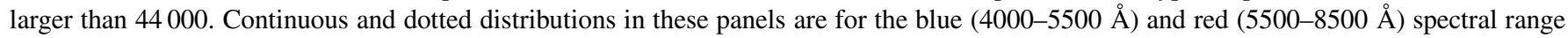
respectively. Panel d) gives the histogram of the number of sight lines at a given redshift in which $\mathrm{Mg}$ II and Fe II absorption lines are in the observed wavelength range. The 18 sight lines in our sample cover the redshift range between 1 and 2 uniformly.

recovers the input value of $\Delta \alpha / \alpha$ that is used while generating the spectrum.

\subsection{Generation of absorption lines}

In this section, we simulate data for different assumed values of $\Delta \alpha / \alpha$ and apply our procedure to recover the input value of $\Delta \alpha / \alpha$. We mainly concentrate on absorption lines from the $\mathrm{Mg}$ II doublet and Fe II multiplets. We assume the ratio, $N(\mathrm{Mg}$ II $) / N(\mathrm{Fe}$ II $)$, to be constant in individual components. In order to mimic real data, the Voigt profile of the absorption line is convolved with an instrumental profile and appropriate noise is added to the spectrum. We model the instrumental profile as a single Gaussian with $\sigma=1.69$ pixels (with pixel size of $0.03 \AA$ ) appropriate for $\lambda / \Delta \lambda=42000$ as achieved in a typical UVES observation (note however that the actual resolution achieved is somewhat higher in the real data).
The noise in the spectrum is the combination of photon noise, readout noise, and the residuals from background subtraction. While the former dominates the error in the continuum the latter two will be the source of error in the core of saturated lines. We notice from our UVES data that the root mean square of the residual at the bottom of highly saturated lines is $\simeq 0.4 \%$ of the unabsorbed continuum flux. The readout noise added to a typical normalized spectrum mimics a Gaussian distribution with zero mean and a standard deviation of $4 \times 10^{-3}$. The photon noise spectrum is obtain using Poisson statistics. To obtain a spectrum with a given signal-to-noise ratio, $(S / N)$, we have generated Poisson random numbers $\left(P\left[(S / N)^{2}\right]\right)$, with a mean equal to $(S / N)^{2}$, and then assigned photon noise to the continuum of the normalized spectrum to be $\left(P\left[(S / N)^{2}\right]-(S / N)^{2}\right) /(S / N)^{2}$, having a Poisson distribution with $\sigma=(S / N)^{-1}$. In the absorbed portion of the spectrum this value is scaled by the square root of the intensity. In all our simulations we assume $S / N$ in the 

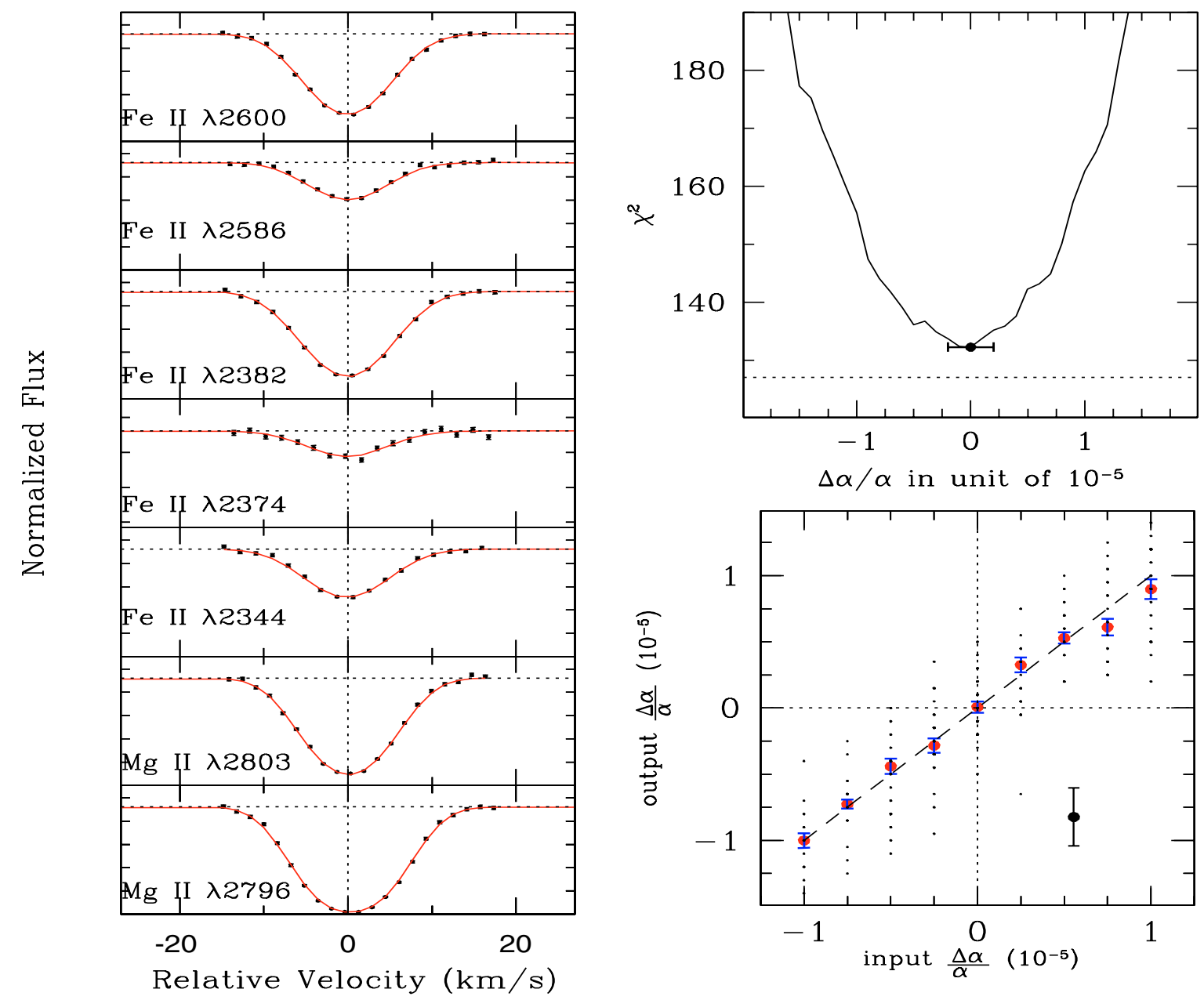

$\Delta \alpha / \alpha$ in unit of $10^{-5}$

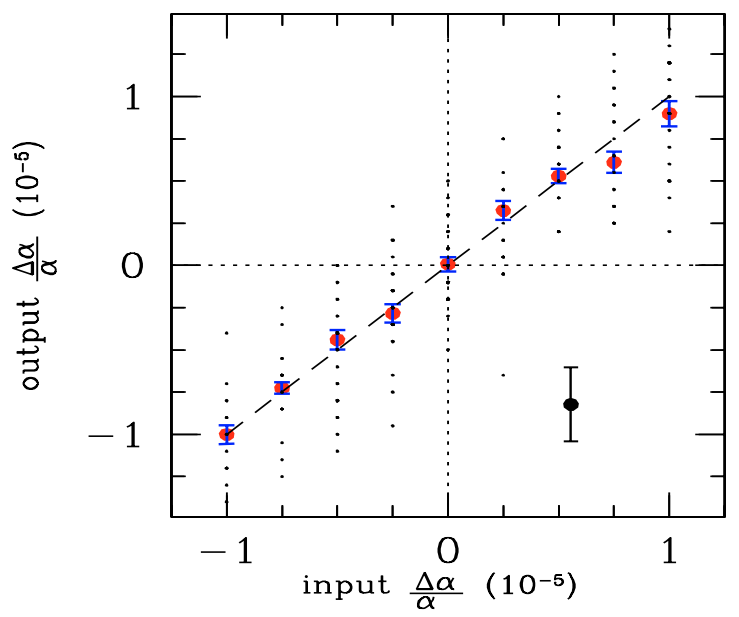

Fig. 2. Illustration of the methodology and reliability of our Voigt profile-fitting code: the panels on the left-hand side show the velocity plot of the simulated Mg II and Fe II absorption lines (points with error bars) and the best-fit Voigt profiles (solid curve). The panel at the top right-hand side shows the $\chi^{2}$ as a function of $\Delta \alpha / \alpha$. The best-fit value and $1 \sigma$ error are shown in the panel. This is consistent with the input value of $\Delta \alpha / \alpha=0$. The lower right-hand panel shows the relationship between the input and recovered values of $\Delta \alpha / \alpha$. Each point is a result obtained from a single realization (there are 30 of them for a given input $\Delta \alpha / \alpha$ ). Typical errors in these measurements are shown in the bottom right corner of the panel. The dark circles with error bars represent the weighted mean value of $\Delta \alpha / \alpha$ obtained from 30 realizations.

continuum to be 70 , corresponding to what is achieved in our data.

To simulate a spectrum incorporating a varying finestructure constant $\left(\alpha_{z}\right)$, we use the analytic fitting function given by Dzuba et al. (2002),

$\omega=\omega_{0}+q x$.

Here $\omega_{0}$ and $\omega$ are, respectively, the vacuum wave number (in unit of $\mathrm{cm}^{-1}$ ) measured in the laboratory and in the absorption system at redshift $z, x$ is a dimensionless number defined by $x=\left(\alpha_{z} / \alpha_{0}\right)^{2}-1$ where $\alpha_{0}$ refers to the present value of the finestructure constant and $\alpha_{z}$ to its value at redshift $z$. The numerical values of the parameter $q$ that are obtained using many-body relativistic calculations (see Dzuba et al. 1999, 2002) for different species are listed in the fifth column of Table 1. Originally $\omega$ is defined as $\omega=\omega_{0}+q_{1} x+q_{2} y$ with $y=\left(\alpha_{z} / \alpha_{0}\right)^{4}-1$ and $x$ as defined above (Dzuba et al. 1999). When $\Delta \alpha / \alpha$ is small $q$ is basically $q_{1}+2 q_{2}$. The table also gives the laboratory values of wavelengths (with standard isotopic composition for different elements) $\left(\lambda_{0}\right)$, wavenumbers $\left(\omega_{0}\right)$ and oscillator strengths $(f)$ used in this study. References to various parameters used are also provided in this table.

\subsection{Voigt profile fitting code}

To measure the relative shifts between an anchor and Fe II lines, we fit all the lines simultaneously. In addition, we have modified our Voigt profile fitting code (Khare et al. 1997) to take into account the dependence of rest wavelengths of different species on $\alpha$. We improved the convolution algorithm using a 41-point Gaussian quadrature integration scheme. The latter is crucial for fitting narrow lines in high signal-to-noise data. The $F W H M$ of the Gaussian kernel is considered to be $\lambda_{\mathrm{r}} / R$. Here $\lambda_{\mathrm{r}}$ is the median wavelength over the region of the absorption line being fitted and $R$ is the spectral resolution. We have taken a $\pm 3 \sigma(\sigma=F W H M / 2.3548)$ wavelength range around each pixel of absorption line for convolution. The Gaussian quadrature integration method is adopted because it provides the most accurate estimate of the area under the Gaussian kernel that is used for normalizing the truncated Gaussian probability 
function. Our code also takes into account the variation of spectral resolution across the spectrum.

\subsection{Measurement of $\Delta \alpha / \alpha$ in a single component systems}

In order to find the best $\Delta \alpha / \alpha$ consistent with our data, we fit the absorption lines using the modified rest wavelengths (see Eq. (1)), varying $\Delta \alpha / \alpha$ in the range from $-5.0 \times 10^{-5}$ to $5.0 \times 10^{-5}$ in steps of $0.1 \times 10^{-5}$. For each value we obtain $\chi_{\min }^{2}$ by varying $N, b$ and $z$. The value of $\Delta \alpha / \alpha$ at which $\chi_{\min }^{2}$ is minimum is accepted as the best possible $\Delta \alpha / \alpha$ value, provided the reduced $\chi^{2}$ of fit is $\simeq 1$. Following the standard statistical procedure (Press et al. 2000, see pages 690-691) we assign $1 \sigma$ errors to the above defined best value of $\Delta \alpha / \alpha$ by computing the required change in $\Delta \alpha / \alpha$ so that $\Delta \chi^{2}=\chi^{2}-\chi_{\min }^{2}=1$. To be on the conservative side we have taken the maximum change in $\Delta \alpha / \alpha$ around the best value of $\Delta \alpha / \alpha$ that is required to produce $\Delta \chi^{2}=1$ as our error on $\Delta \alpha / \alpha$.

We test the method discussed above using a simulated single component system (see Fig. 2). The profiles of Mg II and Fe II absorption lines that are generated for $\Delta \alpha / \alpha=0$ are shown on the left-hand panels of the figure. In the top right-hand panel we show the best fitted $\chi^{2}$ as a function of $\Delta \alpha / \alpha$. The location of the minimum and $1 \sigma$ error in $\Delta \alpha / \alpha$ are shown using a point with an error bar. To investigate the systematics in detail, we have simulated single component systems with column densities in the range $10^{13}$ to $10^{16} \mathrm{~cm}^{-2}$ (we consider that the number of systems with a given $N(\mathrm{Mg}$ II) is proportional to $N(\mathrm{Mg} \mathrm{II})^{-1.4}$, Srianand \& Khare 1994$)$, the velocity dispersion uniformly distributed in the range 3 to $10 \mathrm{~km} \mathrm{~s}^{-1}$ and the input $\Delta \alpha / \alpha$ varying between $-10^{-5}$ and $+10^{-5}$. In addition we have varied the seed for the random number generator that produces the error spectrum. We use our Voigt profile fitting code to recover $\Delta \alpha / \alpha$ in each case. The recovered value of $\Delta \alpha / \alpha$ in each realization is plotted (dots) as a function of the input $\Delta \alpha / \alpha$ in the lower right-hand panel in Fig. 2. The typical error in an individual measurement is also shown in the plot. There are about 30 realizations for a given value of input $\Delta \alpha / \alpha$ with a varied $N, b$ and noise spectrum. The dark circles with error bars represent the weighted mean value of the output $\Delta \alpha / \alpha$ using all the 30 measurements. The weighted mean recovered values of $\Delta \alpha / \alpha$ are consistent with the input values.

It is clear from Fig. 2 that our procedure works well for simple single-component systems. We notice that the distribution of differences between $\Delta \alpha / \alpha$ output and input values is well fitted by a Gaussian (see Fig. 5) with $\sigma=0.23 \times 10^{-5}$. Based on the central limit theorem and a Gaussian distribution of the recovered values around the mean we expect the accuracy of the estimated mean value to be $\sim \sigma / \sqrt{N}$ for $N$ measurements. Strong blending and internal structure in the cloud will imply larger uncertainty.

The improvement we expect from the enhanced $S / N$ ratio in our sample compared to other studies is visualized in Fig. 3. Here, we show the results obtained for individual realizations of single-component systems with input $\Delta \alpha / \alpha=0$ for $S / N=30$ and 70 in the top and bottom panels respectively. The shaded

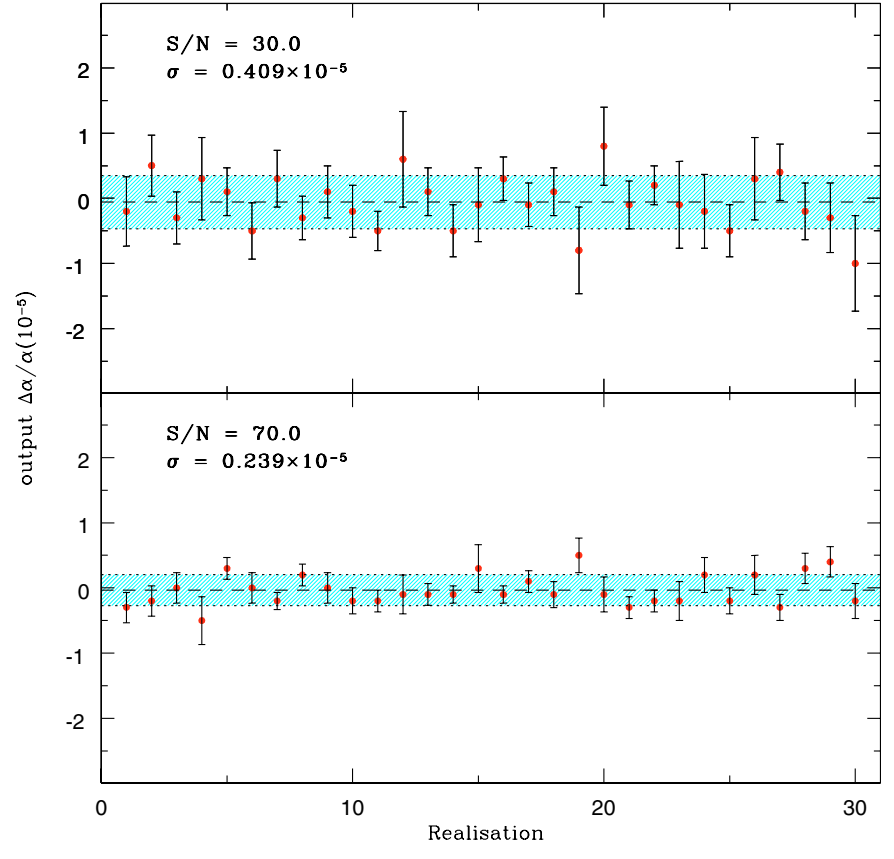

Fig. 3. The effect of signal-to-noise ratio: the figure shows the measured $\Delta \alpha / \alpha$ and $1 \sigma$ error bar from 30 realizations of absorption systems all simulated with an input value $\Delta \alpha / \alpha=0$. The shaded region is the standard deviation $(1 \sigma)$ in the measured values around the mean value (shown by the horizontal dashed line). The absorption systems are simulated using random realizations of $N, b$ and noise spectrum. We obtained $\sigma=0.409 \times 10^{-5}$ and $0.239 \times 10^{-5}$ based on 30 realizations with signal-to-noise 30 and 70 respectively. It is apparent from the figure that better $S / N$ will improve the measurements of $\Delta \alpha / \alpha$.

region in the figure shows the mean and $\sigma$ in the distribution of the recovered values. The distribution of individual values has $\sigma$ of $0.409 \times 10^{-5}$ and $0.239 \times 10^{-5}$ for signal-to-noise ratios 30 and 70 respectively.

It is clear that the improvement in the $S / N$ ratio gives a factor 2 improved accuracy in the recovered value compared to what has been done previously. In all the simulations discussed till now we have used only strong but unsaturated Fe II lines (i.e. $N(\mathrm{Fe}$ II $) \geq 10^{13} \mathrm{~cm}^{-2}$ ). This ensures that the absorption profiles are well defined. However, when the lines are weak the flux in the line is appreciably affected by Poisson noise. One can therefore infer that the scatter in the measurements will be larger if weak lines are used in the analysis. We demonstrate this using simulations of weak lines. For simplicity we have assumed input $\Delta \alpha / \alpha=0, N(\mathrm{Mg}$ II $)=1.5 \times 10^{12} \mathrm{~cm}^{-2}$ and $N(\mathrm{Fe}$ II $)=5 \times 10^{11} \mathrm{~cm}^{-2}$. We have simulated 30 systems by just changing the error spectrum. The results are summarized in Fig. 4. In the left-hand panel we show the profiles for one realization. From this figure one can appreciate the distortion in the absorption profile of Fe II lines caused by Poisson fluctuations. The top panel on the right-hand side shows the recovered values of $\Delta \alpha / \alpha$. As expected, the error bars are larger (one $\sigma$ of the distribution around the mean is $2.92 \times 10^{-5}$ ). Typical errors are then a factor 10 higher than what we derive with strong single-component systems. Thus if one is going to use $1 / \sigma^{2}$ weighting of different measurements, only one single strong and narrow component can be as important as about 

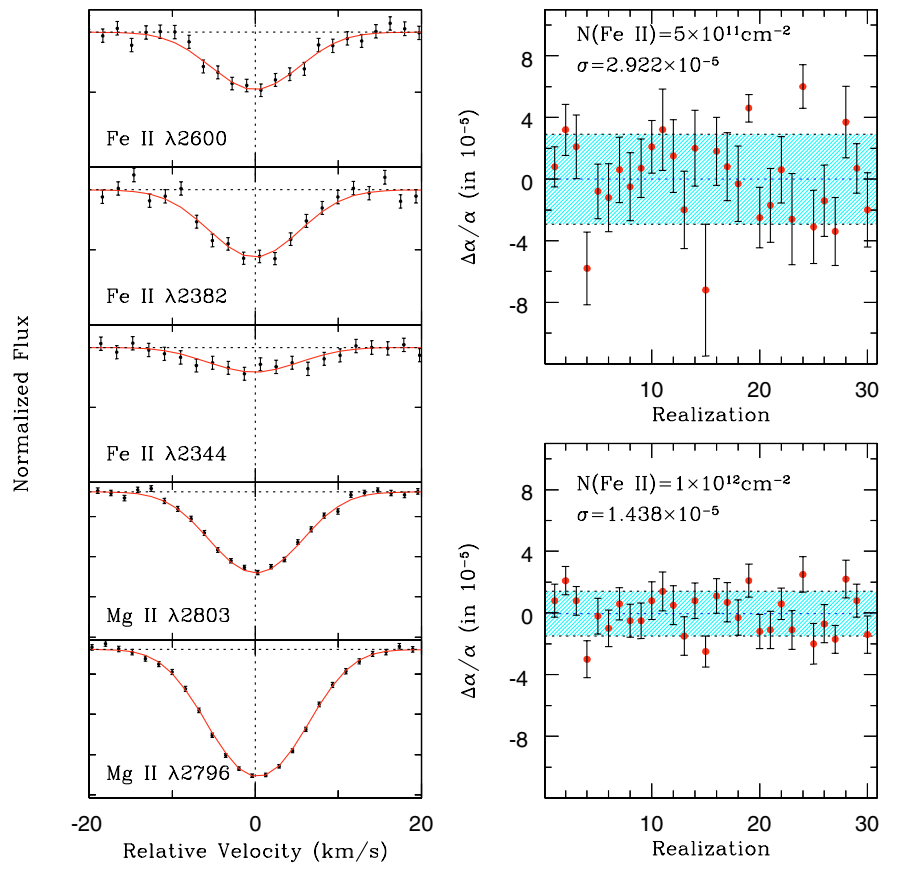

Fig. 4. Simulations of weak lines: the left-hand panels show the velocity plot of the absorption lines simulated in one of the realizations. The best-fit Voigt profiles are overplotted. The right-hand panels give the recovered value of $\Delta \alpha / \alpha$ in individual simulations for two values of $N(\mathrm{Fe}$ II $)$. In all cases the input $\Delta \alpha / \alpha$ is zero and only the error spectrum is changed. The shaded regions in these panels gives the standard deviation around the mean. The numerical values are also given in each panel.

100 weak systems. When considering 30 realizations, we find a weighed mean of $\Delta \alpha / \alpha=(-0.828 \pm 0.508) \times 10^{-5}$ which is a $1.6 \sigma$ deviation with respect to the zero input value. The results shown in the bottom right-hand panel in Fig. 4 are for $N(\mathrm{Fe}$ II $)=10^{12} \mathrm{~cm}^{-2}$ and $N(\mathrm{Mg}$ II $)=3 \times 10^{12} \mathrm{~cm}^{-2}$. The distribution of individual measurements has $\sigma=1.43 \times 10^{-5}$ and the weighted mean value $\Delta \alpha / \alpha=(0.076 \pm 0.273) \times 10^{-5}$. Thus this study clearly demonstrates that if the lines are weak and the profiles are dominated by Poisson errors then a marginally significant false-alarm detection of $\Delta \alpha / \alpha$ can be obtained even when one uses a large number of systems. This can be avoided in the analysis of real data by carefully estimating the appropriate lower limit cutoff in column density for the sample. Based on our simulations and data (see below) we obtain this cutoff for the Fe II column density to be of the order of $2 \times 10^{12} \mathrm{~cm}^{-2}$ for the median $S / N$ ratio corresponding to our data.

\section{4. $\Delta \alpha / \alpha$ measurement in strongly blended systems}

Next we consider the case of strongly blended two-component systems. Here $N$ and $b$ for the two components are chosen in the same way as for the single component systems (see Sect. 3.1). The separation between the two components is taken between 3 and $5 \mathrm{~km} \mathrm{~s}^{-1}$. This means that the subcomponents are separated by less than the width of individual components. The relationship between the input and the recovered $\Delta \alpha / \alpha$ is shown in the top right-hand panel of Fig. 5. This panel also shows a typical simulated profile. There are 30 realizations for a given input $\Delta \alpha / \alpha$. The typical error in the recovered values is also shown in the figure. It is apparent that individual measurements deviate much more from the actual value than they do in the single-component case. Unlike the case of single component systems the deviation with respect to the actual value cannot be approximated by a single Gaussian profile (see bottom right-hand panel in Fig. 5). There is an extra tail in the distribution on both sides. This clearly demonstrates that statistically significant deviations from the real value are possible for a non-negligible fraction of the systems. To avoid this, we will predominantly use systems that are not strongly blended (i.e., for which the sub-component separations are larger than the individual $b$ parameters).

\section{5. $\Delta \alpha / \alpha$ measurements from well separated blends}

The next step is to investigate the case of multicomponent systems where subcomponents are marginally blended. That is the cases where the separation between individual components is larger than the individual $b$ values. Results are summarized in Fig. 6. The left-hand side panel shows velocity profiles for one of the realizations. In the right hand-side panels we compare results obtained for the case considered here (middle panel) with that obtained for single component systems (lower panel) and strongly blended systems (top panel). The $\sigma$ of the distribution around the mean are $0.221 \times 10^{-5}$, $0.261 \times 10^{-5}$ and $0.519 \times 10^{-5}$ for the single, weakly blended and strongly blended systems respectively. It is apparent that the mildly blended case gives very similar results as that obtained with single components.

\subsection{Conclusions from simulations}

In short, we can conclude from these simulations that:

1. Best constraints on $\Delta \alpha / \alpha$ are obtained either from single component systems or well resolved multiple component systems.

2. Increasing the signal-to-noise ratio from $S / N=30$ to 70 increases the accuracy of $\Delta \alpha / \alpha$ measurements by about a factor of two.

3. It is better to avoid weak lines while extracting $\Delta \alpha / \alpha$, as their profiles can be distorted by Poisson noise. Thus, weak lines in the low-signal-to-noise data can result in falsealarm detections of non-zero $\Delta \alpha / \alpha$ values.

4. There is a non-negligible probability for deriving a statistically significant deviation from the actual value when one considers highly blended systems (i.e., systems where the component separations are smaller than the individual $b$ values). Thus it is better to avoid complex blends in the analysis.

In what follows we use these results as guidelines for choosing the systems in our sample for the final analysis. 

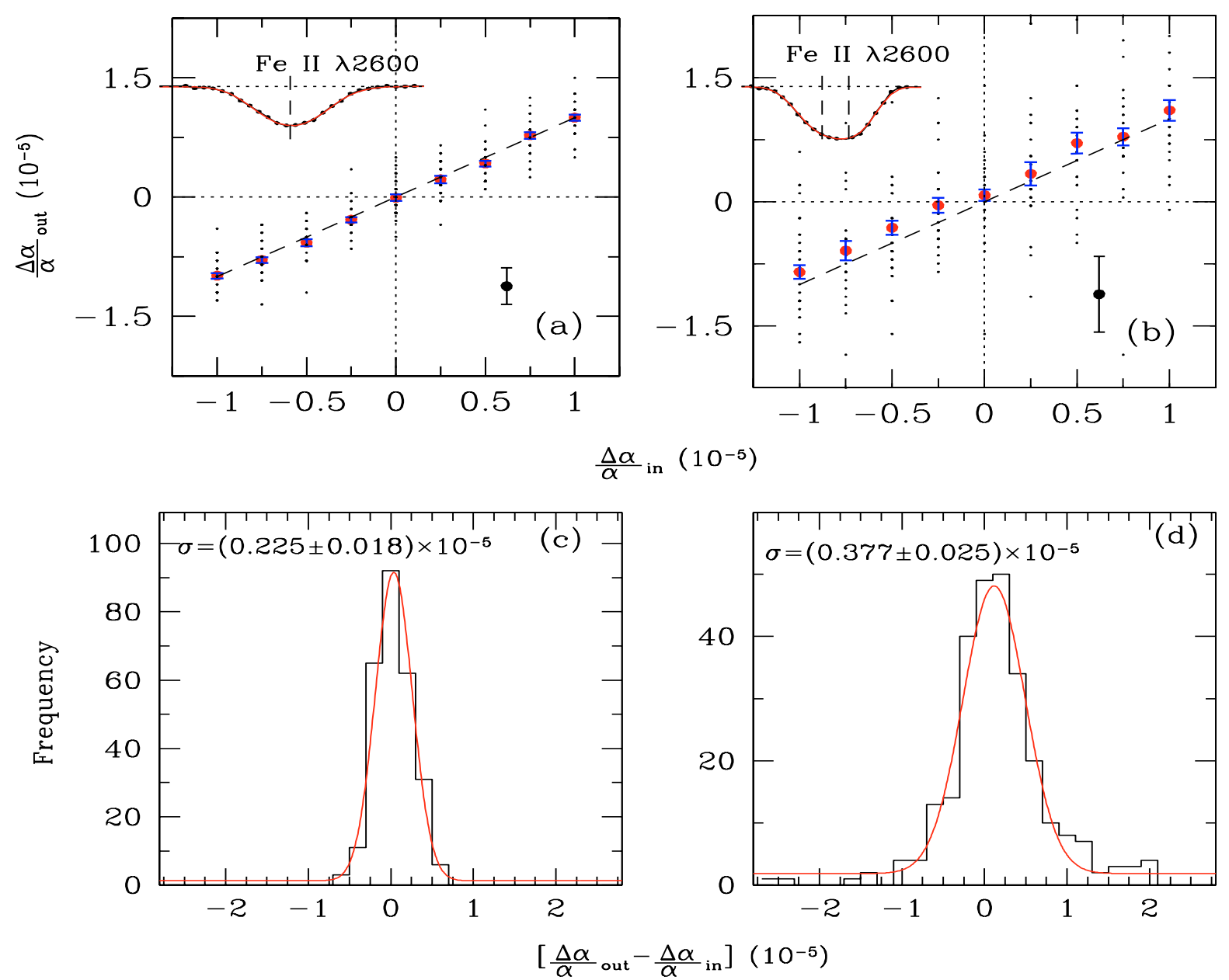

Fig. 5. Effect of blending in the determination of $\Delta \alpha / \alpha$ : absorption spectra of Mg II and Fe II were simulated for given $N, b$, and spectral resolution similar to that of our data, introducing spectral shifts corresponding to a given value of $\Delta \alpha / \alpha$. The top panels show the relationship between the input and derived value of $\Delta \alpha / \alpha$ in the case of a single clean component (left-hand side) and a blend of two components (right-hand side). A typical absorption profile is also shown in these panels. Dots are the values from individual realizations; the points with the error bars represent the weighted mean over 30 realizations. The lower panels give the distribution of the recovered $\Delta \alpha / \alpha$ around the true one. Single (left) and blended (right) cases are considered respectively. Fits by Gaussian distributions are over-plotted.

\section{Description of the UVES sample}

The Mg II systems with detectable Fe II absorption lines present in our data are listed in Table 2. The table provides the QSO name, the emission redshift $\left(z_{\mathrm{em}}\right)$, the redshift of the absorption line systems $\left(z_{\text {abs }}\right)$ and the species that are detected (notations are as in Table 1). No such Mg II systems are detected along 2 sight lines (toward HE 1158-1843 at $z_{\mathrm{em}}=2.449$ and Q 0420-388 at $z_{\mathrm{em}}=3.177$ ). The last column of the table is meant for comments on the systems.

In total, there are 50 systems with detected $\mathrm{Mg}$ II and Fe II lines at a detection limit of $\sim 1.5 \times 10^{11} \mathrm{~cm}^{-2}$ for the strongest Fe II $\lambda 2382$ line. The corresponding limit for Fe II $\lambda 2374$ is $1.5 \times 10^{12} \mathrm{~cm}^{-2}$. In the following we discard systems with $N($ Fe II $)<2 \times 10^{12} \mathrm{~cm}^{-2}$, as simulations have shown that spurious effects can affect the results when the lines are weak. Therefore systems with $N(\mathrm{Fe}$ II) less than this limit (15 systems) are marked as "weak" in Table 2 and are not considered in our main analysis. However, for completeness, we present the results obtained from these systems also in the concluding section.
Two systems at $z_{\mathrm{abs}}=1.0598$ and $z_{\mathrm{abs}}=1.9183$ toward HE 0940-1050 could not be considered in our analysis because there are no good anchor lines in these systems. In the former system only Mg II 12796 is present in the spectrum. In the case of the $z_{\mathrm{abs}}=1.9183$ system, Si II and Al II are blended with Ly $\alpha$ lines and Mg II is heavily affected by atmospheric lines. In addition, at $z_{\mathrm{abs}}=0.726176$ toward Q $0453-423, z_{\mathrm{abs}}=0.8367$ toward Q 0002-422 and $z_{\mathrm{abs}}=0.6631$ toward PKS 2126-158, the Fe II lines are heavily blended with Ly $\alpha$ systems and are therefore marked as "Ly $\alpha$ ". Thus we are left with 29 systems that have strong Fe II lines, good anchor lines and are not contaminated by Ly $\alpha$ or atmospheric absorption. These systems form the basic dataset that we use for measurement of $\Delta \alpha / \alpha$.

In summary, based on the results from the simulations, we apply the following selection criteria to derive reliable $\Delta \alpha / \alpha$ :

1. We consider only lines with similar ionization potentials ( $\mathrm{Mg}$ II, Fe II, Si II and Al II) as they are most likely to originate from similar regions in the cloud.

2. We avoid absorption lines that are contaminated by atmospheric lines. 
Table 2. List of Mg II and Fe II systems.

\begin{tabular}{|c|c|c|c|c|}
\hline QSO & $z_{\mathrm{em}}$ & $z_{\text {abs }}$ & Transitions $^{p}$ & Comments \\
\hline \multirow[t]{3}{*}{ HE 1341-1020 } & 2.135 & 0.8728 & abdefghi & \\
\hline & & 1.2778 & abcdefghi & \\
\hline & & 1.9154 & abcdefghi & \\
\hline \multirow[t]{4}{*}{ Q 0122-380 } & 2.190 & 0.8225 & abdfghi & \\
\hline & & 0.8585 & abefgh & \\
\hline & & 1.2433 & abdefi & \\
\hline & & 1.9102 & abfh & weak \\
\hline PKS 1448-232 & 2.220 & 1.5847 & abfh & weak \\
\hline \multirow[t]{5}{*}{ PKS 0237-23 } & 2.222 & 1.1846 & abfgh & weak \\
\hline & & 1.3650 & abcdefghi & \\
\hline & & 1.6371 & abcdefghi & \\
\hline & & 1.6575 & abcdefghi & \\
\hline & & 1.6724 & abcdefghi & \\
\hline \multirow[t]{5}{*}{ HE 0001-2340 } & 2.263 & 0.4524 & abdefghi & \\
\hline & & 0.9489 & abfh & weak \\
\hline & & 1.5855 & abicdefgh & \\
\hline & & 1.6517 & abfi & weak \\
\hline & & 2.1839 & abdefghi & \\
\hline \multirow[t]{2}{*}{ Q 0109-3518 } & 2.404 & 1.1828 & abdfghi & \\
\hline & & 1.3499 & abcdefghi & \\
\hline \multirow[t]{5}{*}{ HE 2217-2818 } & 2.414 & 0.7862 & abfh & weak \\
\hline & & 0.9425 & abdfghi & \\
\hline & & 1.6917 & abcdefghi & \\
\hline & & 1.6277 & abcdefghi & weak \\
\hline & & 1.5556 & abcdfghi & \\
\hline \multirow{2}{*}{ Q 0329-385 } & 2.435 & 0.7627 & abefghi & \\
\hline & & 1.4379 & abdhi & weak \\
\hline \multirow[t]{2}{*}{ HE $1347-2457$} & 2.611 & 1.4392 & abcdefghi & \\
\hline & & 1.5082 & abdfh & weak \\
\hline \multirow{7}{*}{ Q 0453-423 } & 2.658 & 0.7261 & abdefghi & $\operatorname{Ly} \alpha$ \\
\hline & & 0.9083 & abdefghi & \\
\hline & & 1.0394 & $\mathrm{abh}$ & weak \\
\hline & & 1.1492 & abdefghi & + \\
\hline & & 1.6302 & abf & weak \\
\hline & & 1.8584 & abdfh & \\
\hline & & 2.3004 & abcdef & + \\
\hline PKS 0329-255 & 2.703 & 0.9926 & abdfghi & weak \\
\hline \multirow[t]{5}{*}{ Q 0002-422 } & 2.767 & 0.8367 & abdefghi & $\operatorname{Ly} \alpha$ \\
\hline & & 1.5418 & abdefghi & \\
\hline & & 1.9888 & abdefghi & weak \\
\hline & & 2.1678 & abcdefghi & \\
\hline & & 2.3018 & abcdefi & \\
\hline \multirow[t]{2}{*}{ HE $0151-4326$} & 2.789 & 0.6632 & abdefghi & + \\
\hline & & 1.7319 & $a b f$ & weak \\
\hline HE 2347-4342 & 2.871 & 1.7962 & abdfgh & weak \\
\hline \multirow[t]{3}{*}{ HE 0940-1050 } & 3.084 & 1.0598 & abdgh & no anchor \\
\hline & & 1.7893 & abdefghi & + \\
\hline & & 1.9182 & adefghi & no anchor \\
\hline \multirow[t]{2}{*}{ PKS 2126-158 } & 3.280 & 0.6631 & abdefg & $\operatorname{Ly} \alpha$ \\
\hline & & 2.0225 & abdefgh & \\
\hline
\end{tabular}

${ }^{p}$ Transitions as identified in Table 1.

"weak" refers to systems with $N(\mathrm{Fe}$ II $)<2 \times 10^{12} \mathrm{~cm}^{-2}$.

"+" indicates systems that are not used in the analysis (see discussion).

"Ly $\alpha$ " refers to systems which are blended in the Ly $\alpha$ forest.

"no anchor" refers to systems without good anchor lines.
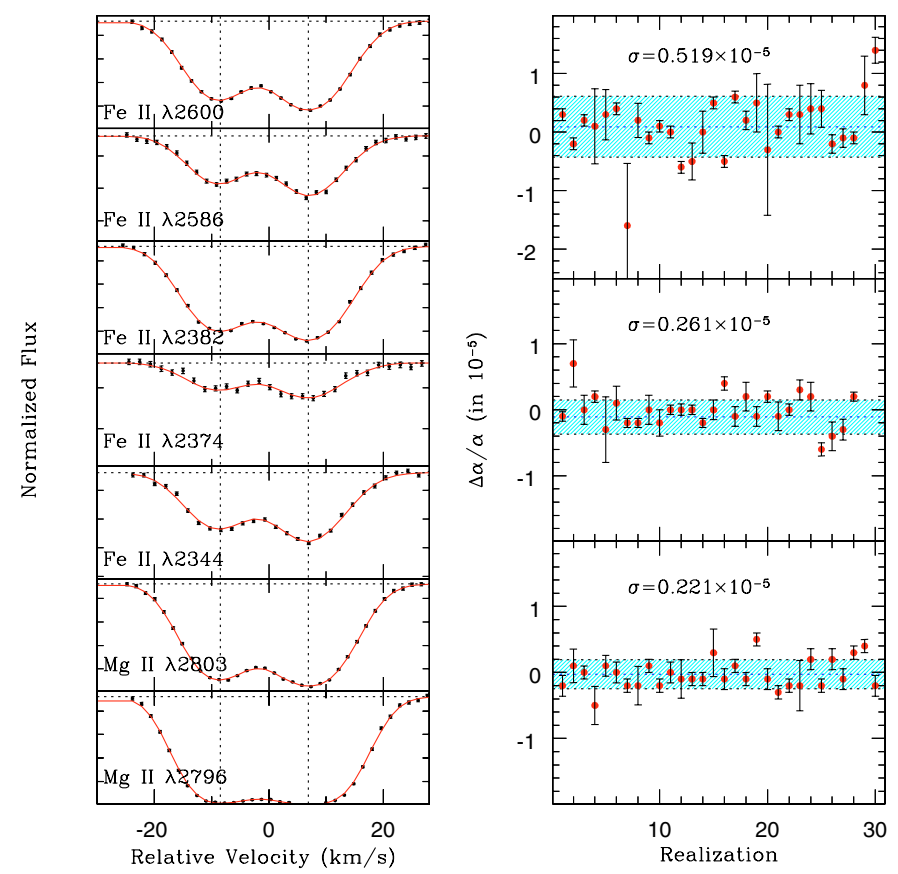

Fig. 6. Results for well-separated blends: left-hand-side panels show the velocity plot and the best-fit Voigt profiles to $\mathrm{Mg}$ II and Fe II absorption lines from one of the realizations. In all cases considered here the velocity separation between the components is always more than the individual values of the $b$ parameters. In the right-hand-side panels we compare the results obtained for heavily blended systems (top panel), well separated blends (middle panel) and single-component systems (lower panel). The standard deviation measured around the mean in different cases is also given in the corresponding panels. It is clear that systems with well separated sub-components have similar $\sigma$ as that of single-component systems for $\frac{\Delta \alpha}{\alpha}$ measurement.

3. We consider only systems that have $N(\mathrm{Fe}$ II $) \geq 2 \times$ $10^{12} \mathrm{~cm}^{-2}$ which ensures that all the standard Fe II multiplets are detected at more than $5 \sigma$ level.

4. We demand that at least one of the anchor lines is not saturated so that the redshift measurement is robust.

5. We also avoided sub-damped Ly $\alpha$ (i.e. $N(\mathrm{H} \mathrm{I}) \geq 10^{19} \mathrm{~cm}^{-2}$. Here after we denote them as sub-DLAs) as these systems may have ionization and chemical inhomogeneities.

6. We do not consider strongly saturated systems with large velocity spread (complex blends); however in such systems whenever we find a well detached satellite components we include these components in the analysis.

7. Finally, based on the component structure resulting from the Voigt profile fits of systems that are not complex blends, we retain only systems for which the majority of components are separated from its neighboring components by more than the $b$ parameters.

Application of the above conditions resulted in 23 systems on which to perform the measurement (six single component systems, six well separated two components (doubles), six systems with three components having at least one well detached from the rest, and five systems with more than 3 components). 


\section{Notes on individual systems in our sample}

\subsection{Systems along the line of sight toward HE 1341-1020}

There are $3 \mathrm{Mg}$ II systems with Fe II along this sight line. All these three systems are strong enough to be included in our sample.

\subsection{1. $z_{\mathrm{abs}}=0.8728$ system toward HE $1341-1020$}

The Voigt profile fits to some of the lines from this system are shown in Fig. 7. This system shows Al II, Mg I 12852 , Ca II $\lambda \lambda 3934,3969$ lines in addition to $\mathrm{Mg}$ II and Fe II lines. The $\mathrm{Mg}$ II profile is fitted with 8 components, the three strongest of which show absorption in other species. The Fe II $\lambda 2600$ line is blended with absorption lines from other intervening systems and the Fe II $\lambda 2374$ profile is affected by a bad pixel. In order to avoid unwanted systematics we do not consider these lines for the $\alpha$ measurement analysis. However we use these profiles to check the consistency in the number of components and the level of saturation in each component. The $\mathrm{Al}$ II line is affected by noise and the Ca II lines are too weak to give any meaningful constraints. We therefore use Fe II $\lambda \lambda \lambda 2344,2382,2586, \mathrm{Mg}$ II $\lambda \lambda 2796,2803$ and $\operatorname{Mg}$ I $\lambda 2852$.

\subsection{2. $z_{\mathrm{abs}}=1.2778$ system toward $\mathrm{HE} 1341-1020$}

The absorption profile of this system is spread over $200 \mathrm{~km} \mathrm{~s}^{-1}$. Absorption lines of Mg I, Mg II, Al II, Al III, Fe II, Ca II, Si II, Mn II, Ni II, and Ca II are detected. The core is strongly blended over $100 \mathrm{~km} \mathrm{~s}^{-1}$ but a well detached narrow satellite is seen $\sim 100 \mathrm{~km} \mathrm{~s}^{-1}$ away. We have used Fe II and Mg II absorptions from this sharp component for the measurement of $\Delta \alpha / \alpha$. We do not use Mn II, Ni II and Ca II because they are weak. Si II and Al II lines fall in the Ly $\alpha$ forest and the profile of Fe II $\lambda 2586$ is affected by the wing of a contaminating neighboring line. The satellite component is well fitted with two components (see middle panel in Fig. 7). Even though the two components are merged, we notice that the components are separated by more than the velocity dispersion of the individual component.

\subsection{3. $z_{\text {abs }}=1.9154$ system toward HE $1341-1020$}

This system shows absorption lines from $\mathrm{Mg}$ II, Mg I, Al II, Al III, C IV, Si II, and Si IV. The high-ionization lines are weak and their profiles are very different from that of the lowionization lines. The $\mathrm{Mg}$ II lines are saturated and blended with atmospheric absorption lines. Thus, we use Si II $\lambda 1526$, Si II $\lambda 1808$ and Fe II lines for $\Delta \alpha / \alpha$ measurement. We do not use Fe II $\lambda 2586$ however as its profile is affected by noise. The system is fitted with 4 distinct components that are clearly visible in the profiles of the weak lines (see right panel in Fig. 7). Even though the overall fit is good, one can notice that the Si II $\lambda 1808$ line profile is apparently not reproduced well. This is due to the effect of noise in a single pixel in the red wing of
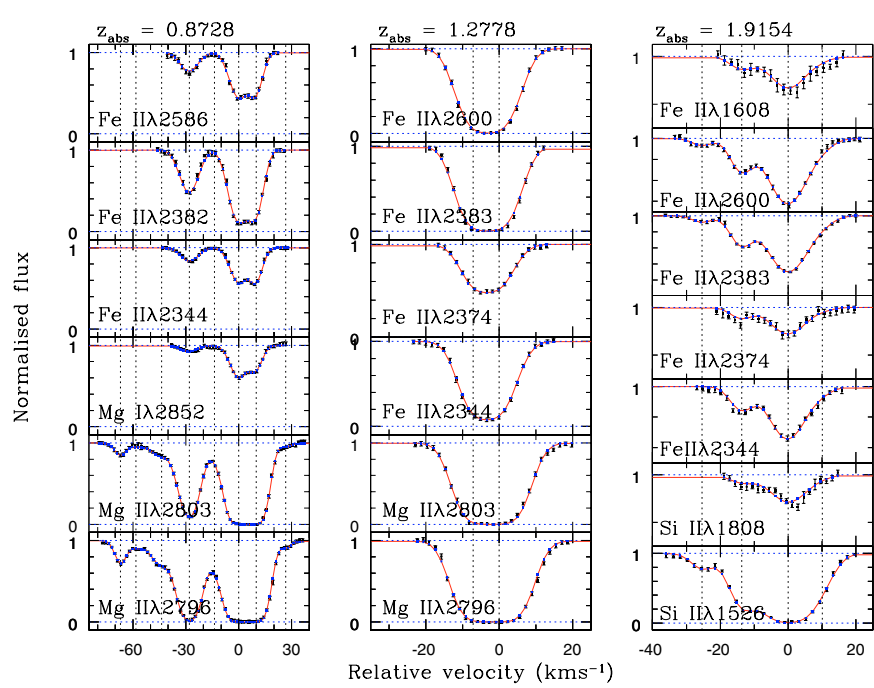

Fig. 7. Voigt profile fits to the absorption systems seen along the line of sight toward $z_{\mathrm{em}}=2.135$ QSO HE 1341-1020. The vertical dotted lines mark the locations of subcomponents.

the strongest absorption component. This shows how delicate the measurement can be.

\subsection{Systems along the line of sight toward Q 0122-380}

There are $4 \mathrm{Mg}$ II systems along this line of sight. The system at $z_{\mathrm{abs}}=1.9102$ has weak Fe II absorption lines and does not fulfill our criteria to enter our main sample.

\subsection{1. $z_{\mathrm{abs}}=0.8221$ system toward $\mathrm{Q} 0122-380$}

This system is defined by narrow $\mathrm{Mg}$ II, Mg I and Fe II absorption lines. All the lines are well fitted by a single component. An extra component in the red wing is required for the $\mathrm{Mg}$ II doublets. The Voigt profile fits to the system are shown in Fig. 8.

\subsection{2. $z_{\mathrm{abs}}=0.8593$ system toward $\mathrm{Q} 0122-380$}

This system is defined by $\mathrm{Mg}$ II, Mg I, Al II, Al III, Ca II, and $\mathrm{Fe}$ II absorption lines. As the spectral range in which Al II absorption is redshifted is of lower signal-to-noise and $\mathrm{Ca}$ II doublets and Fe II $\lambda 2374$ are weak we have not used these lines for the $\Delta \alpha / \alpha$ analysis. The resulting Voigt profile fits are shown in Fig. 8. We notice that the Fe II $\lambda 2586$ line is slightly under-predicted in our best-fit model. However the other three Fe II lines are very well fitted. We also notice that the value of $\chi^{2}$ per degree of freedom is very good when using different $b$ parameters for Fe II and Mg II. The result for $\Delta \alpha / \alpha$ presented for this system is therefore obtained with different $b$ parameters for Mg II and Fe II lines. 

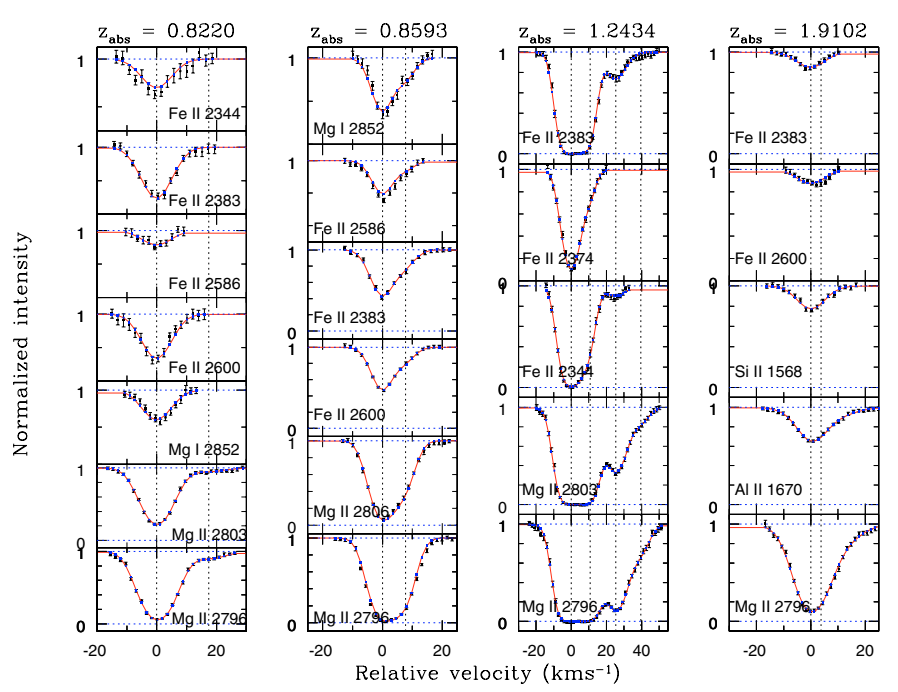

Fig. 8. Voigt profile fits to the four absorption systems seen along the line of sight toward $z_{\mathrm{em}}=2.190$ QSO Q $0122-380$. The vertical dotted lines mark the locations of subcomponents.

\subsection{3. $z_{\mathrm{abs}}=1.2433$ system toward $\mathrm{Q} 0122-380$}

The Mg II absorption profiles in this system are spread over $\sim 70 \mathrm{~km} \mathrm{~s}^{-1}$. The components are heavily blended. The strongest Mg II component shows Mg I, Al III, Si II, Si IV, C IV, $\mathrm{Ca}$ II, Ni II and Fe II. Fe II $\lambda 2586$ and Fe II $\lambda 2600$ lines are not covered by our spectrum. Three Fe II lines and the Mg II doublet can be fitted with 4 components. The Ni II $\lambda 1709$ line is blended and Ni II $\lambda 1741$ is weak and falls in the noisy region of the spectrum. We notice small profile inconsistencies between the $\mathrm{Ca}$ II doublets. We therefore avoid using Ni II and $\mathrm{Ca}$ II lines in the analysis. The results are summarized in Fig. 8.

\subsection{Systems along the line of sight toward PKS 0237-23}

This QSO is known for the presence of a supercluster of $\mathrm{C}$ IV absorption lines at $z_{\text {abs }} \sim 1.6$. Our spectrum reveals $5 \mathrm{Mg}$ II systems one of which at $z_{\mathrm{abs}}=1.1846$ has a very weak Fe II line and is not considered for our main analysis.

\subsection{1. $z_{\text {abs }}=1.3650$ toward PKS 0237-23}

This system shows absorption lines of Fe II, Al II, Al III, C I, C I*, C II, C II*, Si II, Si IV, Ca II, Mg I and Mg II lines. The Ly $\alpha$ line is just below our wavelength coverage. The absorption profiles of strong resonance lines spread over $230 \mathrm{~km} \mathrm{~s}^{-1}$. The main component has a large number of sub-components heavily blended. There is a well detached satellite component that could be used for the $\Delta \alpha / \alpha$ measurements. The presence of C I suggests that this could possibly be a sub-DLA. In such systems inhomogeneities are generic. Thus it is better to avoid them in the $\Delta \alpha / \alpha$ measurements. This system happens to be both in our data and in those of Murphy et al. (2003). Thus we perform the $\Delta \alpha / \alpha$ measurement in the satellite components. We use Al II, all Fe II lines (apart from Fe II 22383), and Mg II lines for our analysis. Murphy et al. (2003) have found $\Delta \alpha / \alpha=-0.19 \pm 0.5$
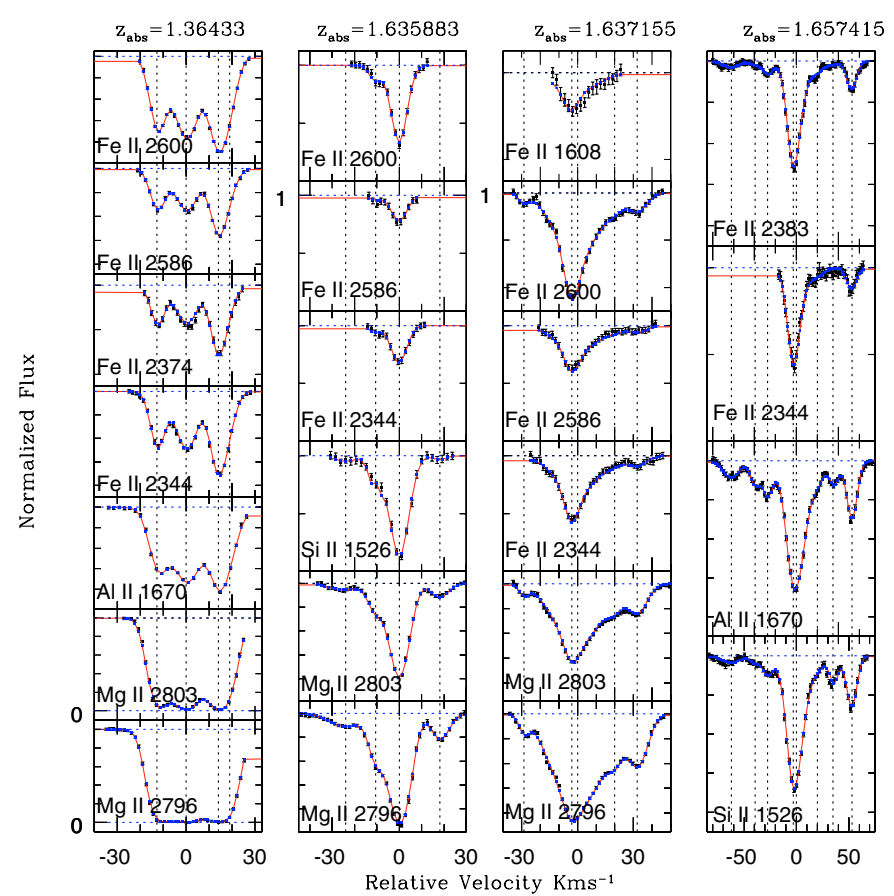

Fig. 9. Voigt profile fits to the five absorption systems seen along the line of sight toward $z_{\mathrm{em}}=2.222$ PKS 0237-23. The vertical dotted lines mark the locations of subcomponents.

for this system. Unlike us Murphy et al. have used the whole profile of Al II, Si II, Al III, and two Fe II lines. Our analysis gives a best-fit value of $0.0 \pm 0.1$. This is consistent with that measured by Murphy et al. and our final result. The increased accuracy in our measurement is contributed to by the enhanced $S / N$ and our choice of the well detached and unblended satellite component for the analysis. For the reason mentioned above we have excluded this system from our analysis.

\subsection{2. $z_{\mathrm{abs}}=1.6371$ toward PKS 0237-23}

The absorption profiles of this system are spread over $\sim 280 \mathrm{~km} \mathrm{~s}^{-1}$. Absorption lines from Mg I, Mg II, Al II, Al III, Si II and Fe II have been detected. Even though C IV and Si IV absorption lines are detected the system is dominated by low-ionization species. There are two distinct velocity components at $z_{\mathrm{abs}}=1.63588$ and 1.63715 that we use for $\Delta \alpha / \alpha$ measurements. The Voigt profile fits to these systems are shown in Fig. 9.

\subsection{3. $z_{\mathrm{abs}}=1.6574$ toward PKS 0237-23}

This system shows absorption lines from $\mathrm{Mg}$ II, Mg I, Al II, Al III, Si II, C IV, Si IV in addition to Fe II. The low- and highionization lines show distinct profiles. C IV and Si IV absorption lines are spread over $400 \mathrm{~km} \mathrm{~s}^{-1}$. We use the uncontaminated Fe II lines with Si II and Al II lines as anchors. The Mg II line is heavily blended and has a large number of extra components compared to Fe II, and Mg I is weak. The results of the Voigt profile fits are shown in Fig. 9. 


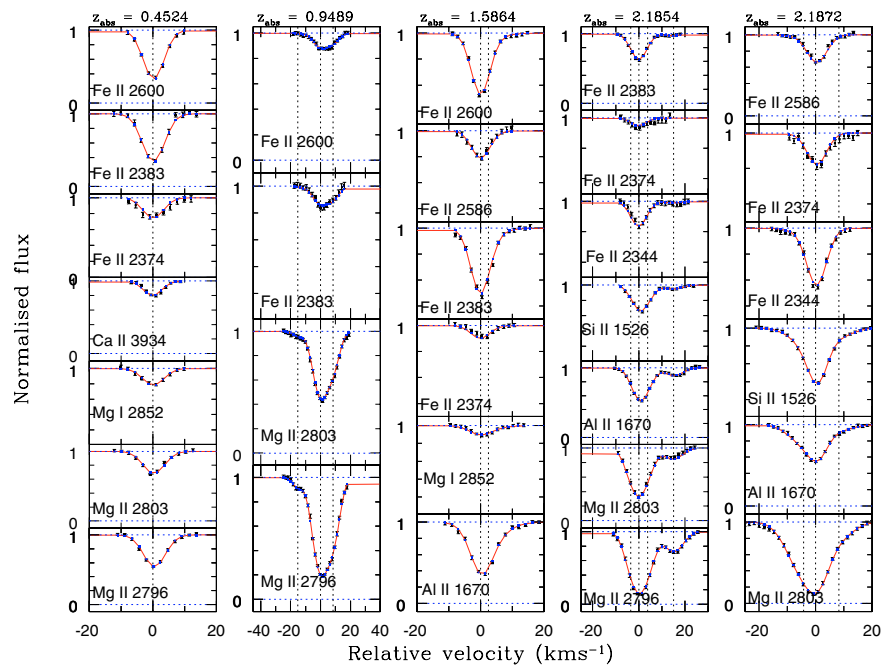

Fig. 10. Voigt profile fits to the five absorption systems seen along the line of sight toward $z_{\mathrm{em}}=2.263$ QSO HE 0001-2340. The vertical dotted lines mark the locations of subcomponents.

\subsection{4. $z_{\mathrm{abs}}=1.6724$ toward PKS 0237-23}

This is one of the few sub-DLAs that are detected in the large programme sample. Mg I, Mg II, C I, C I* C II, C II*, Si II, Si IV, C IV, Ni II, Zn II, Cr II, Mn II and Fe II lines are present. Most of the standard resonance lines are saturated. Zn II, Mn II, Cr II and Ni II absorption lines are weak and therefore will not give good constraints. The presence of the whole range of ionization states and of fine-structure absorption lines strongly suggests strong inhomogeneities in the gas. To keep our analysis unbiased we did not use this sub-DLA system in our analysis.

\subsection{Systems along the line of sight toward HE 0001-2340}

There are $5 \mathrm{Mg}$ II systems detected along this sight line. Two of them at $z_{\mathrm{abs}}=0.9489$ and 1.6517 are weak systems and are not considered in our main sample.

\subsection{1. $z_{\mathrm{abs}}=0.4524$ system toward HE 0001-2340}

This system has two very narrow components separated by $\sim 70 \mathrm{~km} \mathrm{~s}^{-1}$. The low-redshift component shows Mg II, Mg I, Fe II and Ca II $\lambda 3934$ absorption lines. The high-redshift component has very weak Fe II lines. Thus we use only the lowredshift component for $\Delta \alpha / \alpha$ measurement. Voigt profile fits to the absorption lines from this component are shown in Fig. 10. Fe II $\lambda 2344$ and Fe II $\lambda 2586$ are blended with other absorption lines and were not used in the analysis.

\subsection{2. $z_{\mathrm{abs}}=1.5855$ system toward HE 0001-2340}

Like the $z_{\mathrm{abs}}=0.4524$ system this system has two well detached components separated by $\sim 110 \mathrm{~km} \mathrm{~s}^{-1}$. Mg II, Mg I, Fe II, Al II, Al III, C IV, Si II and Si IV absorption lines are seen in both components. However Fe II lines are strong only in the high$z$ component. We notice that Mg II $\lambda 2803$ is contaminated by
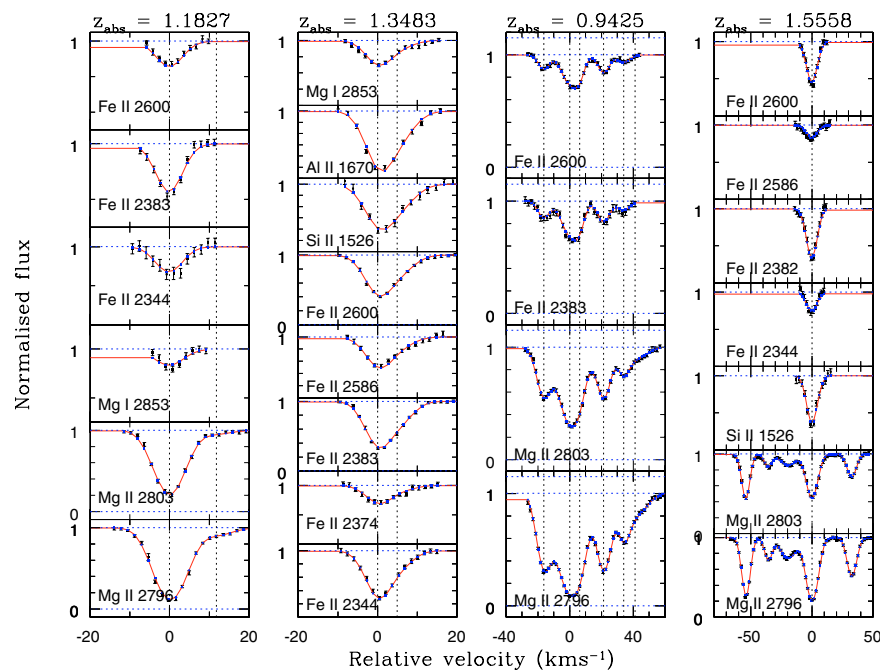

Fig. 11. Voigt profile fits to two of the absorption systems seen along the line of sight toward $z_{\mathrm{em}}=2.404 \mathrm{Q} 0109-3518$ (first and second column from the left) and $z_{\mathrm{em}}=2.414 \mathrm{HE} 2217-2818$ (Cols. 3 and 4 from the left). The vertical dotted lines mark the locations of subcomponents.

an atmospheric line. As Si II $\lambda 1526$ is also blended, we use Al II and Mg I absorption lines as anchors. Even though the overall fit to the data is good, our profiles consistently overpredict the blue wing of Fe II $\lambda 2374$. This is probably due to one single pixel in the wing being affected by noise. As the two components that are required to fit the profiles are very close to each other this system is not considered for the final analysis.

\subsection{3. $z_{\mathrm{abs}}=2.1839$ system toward HE $0001-2340$}

Absorption profiles produced by this system are spread over $400 \mathrm{~km} \mathrm{~s}^{-1}$. Mg I, Mg II, Al II, Al III, Si II, Si IV and C IV absorption lines are detected. The Fe II lines are strong in only two of these components (at $z_{\mathrm{abs}}=2.1854$ and $z_{\mathrm{abs}}=2.1872$ ). As Fe II $\lambda 2383$, Fe II $\lambda 2586$ and Fe II $\lambda 2600$ absorption lines fall in the wavelength range affected by atmospheric absorption, we consider only the clean lines in each case. The results of profile fitting are shown in Fig. 10.

\subsection{Systems along the line of sight toward Q 0109-3518}

There are two Mg II system seen along the line of sight toward this QSO. We used both of them for $\Delta \alpha / \alpha$ measurement.

\subsection{1. $z_{\mathrm{abs}}=1.1827$ system toward $\mathrm{Q} 0109-3518$}

This system shows absorption lines from $\mathrm{Mg}$ II, $\mathrm{Mg}$ I and Fe II. The Fe II and Mg I lines are fitted with a single component but $\mathrm{Mg}$ II requires additional components in the red wing. The results of Voigt profile fitting are shown in Fig. 11. 


\subsection{2. $z_{\mathrm{abs}}=1.3483$ system toward $\mathrm{Q} 0109-3518$}

This is a strong Mg II system with heavily blended absorption profiles spread over $300 \mathrm{~km} \mathrm{~s}^{-1}$. Absorption lines from $\mathrm{Mg} \mathrm{I}$, $\mathrm{Mg}$ II, Fe II, Al II, Al III, Si II, Si IV and C IV are detected. There are two well detached satellite components. We used the $z_{\text {abs }}=$ 1.3483 satellite for $\Delta \alpha / \alpha$ measurement. As $\mathrm{Mg}$ II is blended, Si II, Al II and Mg I lines are used as anchors. For the other satellite the anchor lines are all blended. The results of Voigt profile fitting are presented in Fig. 11.

\subsection{Systems along the line of sight toward $z_{\mathrm{em}}=2.414 \mathrm{HE}$ 2217-2818}

There are five $\mathrm{Mg}$ II systems along the line of sight toward this QSO. Three of these systems (at $z_{\mathrm{abs}}=0.7862,1.6277$ and 1.5556) are weak and are not considered in the main sample. The other two systems were used for $\Delta \alpha / \alpha$ measurement.

\subsection{1. $z_{\text {abs }}=0.9425$ system toward HE 2217-2818}

This system shows absorption lines from $\mathrm{Mg}$ II and Fe II. There are two distinct subsystems that are separated by $\sim 200 \mathrm{~km} \mathrm{~s}^{-1}$. Only the high-redshift subsystem shows Fe II absorption. The Fe II lines are fitted with four well separated components and $\mathrm{Mg}$ II requires an extra component in the red wing. We notice that the goodness of the fit in this system is decided by how well we model the $\mathrm{Mg}$ II profiles. The results of the profile fitting are summarized in Fig. 11.

\subsection{2. $z_{\mathrm{abs}}=1.5556$ system toward HE 2217-2818}

This system shows absorption due to $\mathrm{Mg}$ II, Al II, Si II, $\mathrm{Si}$ IV, C IV and Fe II. The Mg II absorption profiles are spread over $\sim 100 \mathrm{~km} \mathrm{~s}^{-1}$. However only the central velocity component shows other singly ionized species (see Fig. 11). Surprisingly this component also shows absorption due to O I. High-ionization lines such as C IV, Si IV are weak and Al III is absent.

\subsection{Systems along the line of sight toward $z_{\mathrm{em}}=2.435$ QSO Q 0329-385}

There are two absorption systems detected along this line of sight. The $z_{\mathrm{abs}}=1.43799$ system is weak and is not considered in our sample.

\subsection{1. $z_{\text {abs }}=0.7627$ system toward $Q$ 0329-385}

This system shows absorption lines due to $\mathrm{Mg} \mathrm{I}, \mathrm{Mg}$ II, and Fe II. The Mg II absorption profile is spread over $200 \mathrm{~km} \mathrm{~s}^{-1}$. However the other lines are detected only in the central component. The Voigt profile fits to this component are shown in Fig. 12. The profiles are fitted with two components, and as the velocity separation is smaller than the largest $b$ value of the components we do not consider this system in our study.
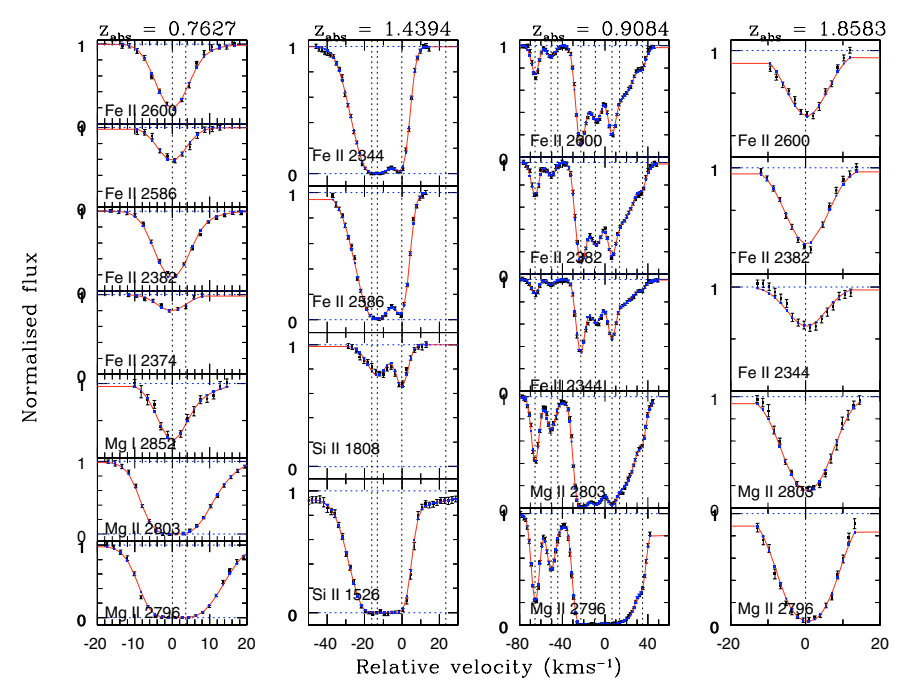

Fig. 12. Voigt profile fits to the absorption systems seen along the line of sight toward $z_{\mathrm{em}}=2.435$ QSO Q 0329-385 (first column from the left), $z_{\mathrm{em}}=2.611$ QSO HE 1347-2457 (Col. 2 from left), and $z_{\mathrm{em}}=$ 2.658 Q 0453-423 (Cols. 3 and 4 from the left). The vertical dotted lines mark the locations of subcomponents.

\subsection{Systems along the line of sight toward Q 0453-423}

There are five systems with detected Fe II and Mg II absorption lines. As most of the Fe II absorption lines of the $z_{\mathrm{abs}}=$ 0.72617 system are redshifted into the wavelength range contaminated by intergalactic Ly- $\alpha \mathrm{H}$ I absorptions we do not use this system for $\Delta \alpha / \alpha$ measurement. The absorption profiles of the $z_{\mathrm{abs}}=1.1492$ system are spread over $400 \mathrm{~km} \mathrm{~s}^{-1}$. The $\mathrm{Mg}$ II and Fe II lines for which we know the $q$ values are all saturated. The profiles of Mg I, Ca II and Mn II suggest a very complex blend. Thus we do not consider this system for $\Delta \alpha / \alpha$ measurement. Finally, the system at $z_{\mathrm{abs}}=1.0394$ show only Fe II $\lambda 2600$ absorption and is therefore not considered in the analysis.

\subsection{1. $z_{\text {abs }}=0.9083$ system along the line of sight toward Q 0453-423}

This system shows detectable absorption from $\mathrm{Mg}$ I, $\mathrm{Mg}$ II, Fe II, Ca II and Al II. Fe II $\lambda 2586$ and Fe II $\lambda 2374$ are contaminated by absorptions from other systems. Based on the Fe II profiles the system is decomposed into eight subcomponents. The profile fits to the $\mathrm{Mg}$ II and Fe II lines free of contamination are shown in Fig. 12.

\subsection{2. $z_{\mathrm{abs}}=1.8583$ system along the line of sight toward Q 0453-423}

This is a narrow single-component absorption system detected in Mg II, Al II, Al III, Si II, Si IV and Fe II. The results of the single-component Voigt profile fit to three prominent Fe II lines and the Mg II doublet are shown in Fig. 12. 


\subsection{3. $z_{\mathrm{abs}}=2.3004$ system along the line of sight toward Q 0453-423}

This system shows absorption lines of Mg II, Fe II and Al II. Fe II $\lambda 2586$ and Fe II $\lambda 2600$ are not covered by our spectrum. Fe II $\lambda 2374$ is very weak. The signal-to-noise ratio in the spectral range where other Fe II lines are seen is $~ 30$ only. In addition, both $\mathrm{Mg}$ II lines are contaminated by atmospheric absorption. There are also profile inconsistencies between the weak Al II lines (a potential anchor) and Fe II lines. Thus we do not consider this system in our study.

\subsection{Absorption systems along the line of sight toward $z_{\mathrm{em}}=2.767 \mathrm{Q} 0002-422$}

There are $5 \mathrm{Mg}$ II systems with detected Fe II absorption lines along this line of sight. The $z_{\mathrm{abs}}=0.8367$ system is a very strong $\mathrm{Mg}$ II system whose absorption profile is spread over $\sim 600 \mathrm{~km} \mathrm{~s}^{-1}$. As the corresponding absorption profiles are heavily blended we do not consider this system in our analysis, as is the case for the $z_{\mathrm{abs}}=1.9885$ system in which Fe II lines are too weak. The remaining 3 systems are considered in our main analysis.

\subsection{1. $z_{\mathrm{abs}}=1.5418$ system toward $\mathrm{Q} 0002-422$}

This system is defined by $\mathrm{Mg}$ II, Mg I, Al II, Al III, and Fe II absorption lines. The Mg II absorption lines are spread over $\sim 180 \mathrm{~km} \mathrm{~s}^{-1}$. The other low-ionization lines are seen only in the strongest central component. Voigt profile fits to the absorption lines are shown in Fig. 13.

\subsection{2. $z_{\mathrm{abs}}=2.1678$ system toward $\mathrm{Q} 0002-422$}

This system is defined by Mg II, Mg I, Al II, Al III, Fe II, Si II, Si IV and C IV absorption lines. Like most systems in our sample the high- and low-ionization profiles are different. The Mg II absorption profile is spread over $80 \mathrm{~km} \mathrm{~s}^{-1}$. However, only the strongest central component shows other low-ionization absorption lines. As the central Mg II component is blended in the red wing with other components we use $\mathrm{Si}$ II and Al II as anchors. The presence of Fe II $\lambda 1608$ with negative $q$ coefficient makes this system very sensitive to $\Delta \alpha / \alpha$. The system is fitted with two components and the velocity separation between them is larger than the individual $b$ values.

\subsection{3. $z_{\mathrm{abs}}=2.3008$ system toward $\mathrm{Q} 0002-422$}

This system is defined by $\mathrm{Mg}$ II, Mg I, Al II, Al III, Fe II, Si II, Si IV and C IV absorption lines. Here also high- and lowionization profiles are different, like most of other systems. The $\mathrm{Mg}$ II absorption profile is spread over $200 \mathrm{~km} \mathrm{~s}^{-1}$. The main component is complex and blended. There is a well detached satellite in the blue wing of the profile. We use this for our $\Delta \alpha / \alpha$ measurement. We also use the unblended Fe II lines and Si II $\lambda 1526$ and Al II lines for our analysis. The three components that fit the profile are well resolved.
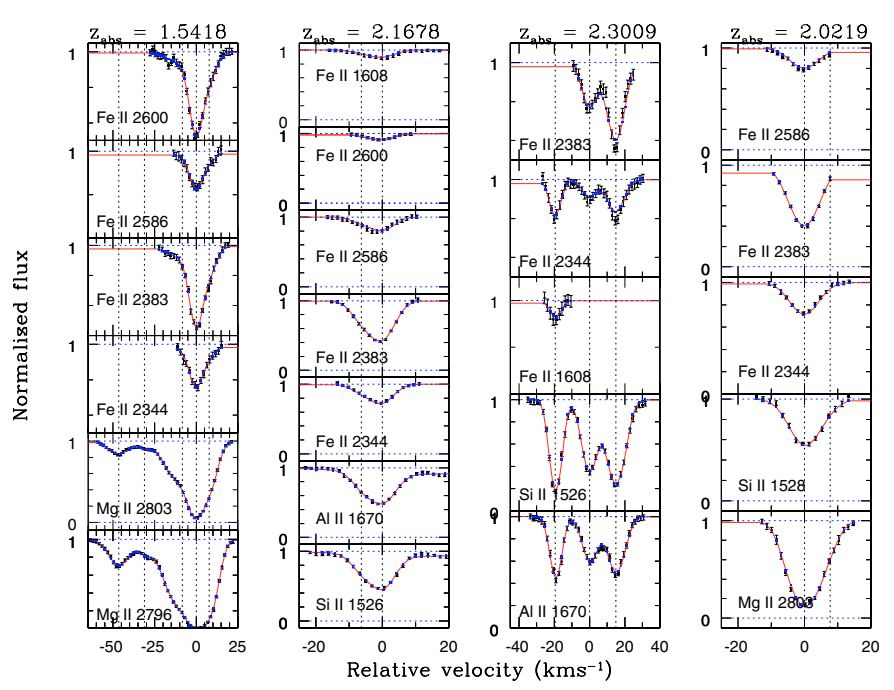

Fig. 13. Voigt profile fits to two of the absorption systems seen along the line of sight toward $z_{\mathrm{em}}=2.767 \mathrm{Q} 0002-422$ (first three columns from the left) and $z_{\mathrm{em}}=3.280$ QSO PKS 2126-158 (last column). Vertical dotted lines mark the locations of subcomponents.

\subsection{Absorption systems along the line of sight toward $z_{\mathrm{em}}=3.280$ QSO PKS 2126-158}

Two Mg II systems are detected along the line of sight toward this QSO. The $z_{\mathrm{abs}}=0.6631$ system shows absorption lines from Mg II, Mg I, Fe II, and Ca II. All the lines are heavily blended with intervening $\operatorname{Ly} \alpha$ absorption lines owing to the large redshift of the QSO. We do not consider this system for our analysis.

\subsection{1. $z_{\text {abs }}=2.0219$ system toward PKS 2126-158}

This system shows absorption due to $\mathrm{Mg}$ II, Si II and Fe II. The Mg II profile is spread over $140 \mathrm{~km} \mathrm{~s}^{-1}$. The strongest $\mathrm{Mg}$ II component is saturated. We use the narrow satellite component on the low redshift side, at $z_{\mathrm{abs}}=2.02192$ for $\Delta \alpha / \alpha$ measurement. As the blue wing of Mg II $\lambda 2796$ is affected by atmospheric absorption, we use Si II and Mg II $\lambda 2803$ as anchors. Voigt profile fits to the lines used in the analysis are shown in Fig. 13.

\section{Results}

\subsection{Fitting procedure}

As discussed before, the accuracy of the method depends on how well absorption line profiles are modeled using Voigt profiles. As can be seen from Table 1 the relative shifts between different Fe II lines (apart from Fe II 21608 ) are small compared to the shifts between the Fe II lines and the anchors. We use this fact to refine our fitting procedure.

We first fit all the Fe II lines simultaneously using laboratory wavelengths $(\Delta \alpha / \alpha=0)$. This allows us to find out about (i) bad pixels, (ii) unknown contaminations and (iii) the velocity component structure in Fe II. We therefore can remove inadequate profiles (because of contamination or bad pixels) from the $\Delta \alpha / \alpha$ analysis. A similar exercise was carried out for 

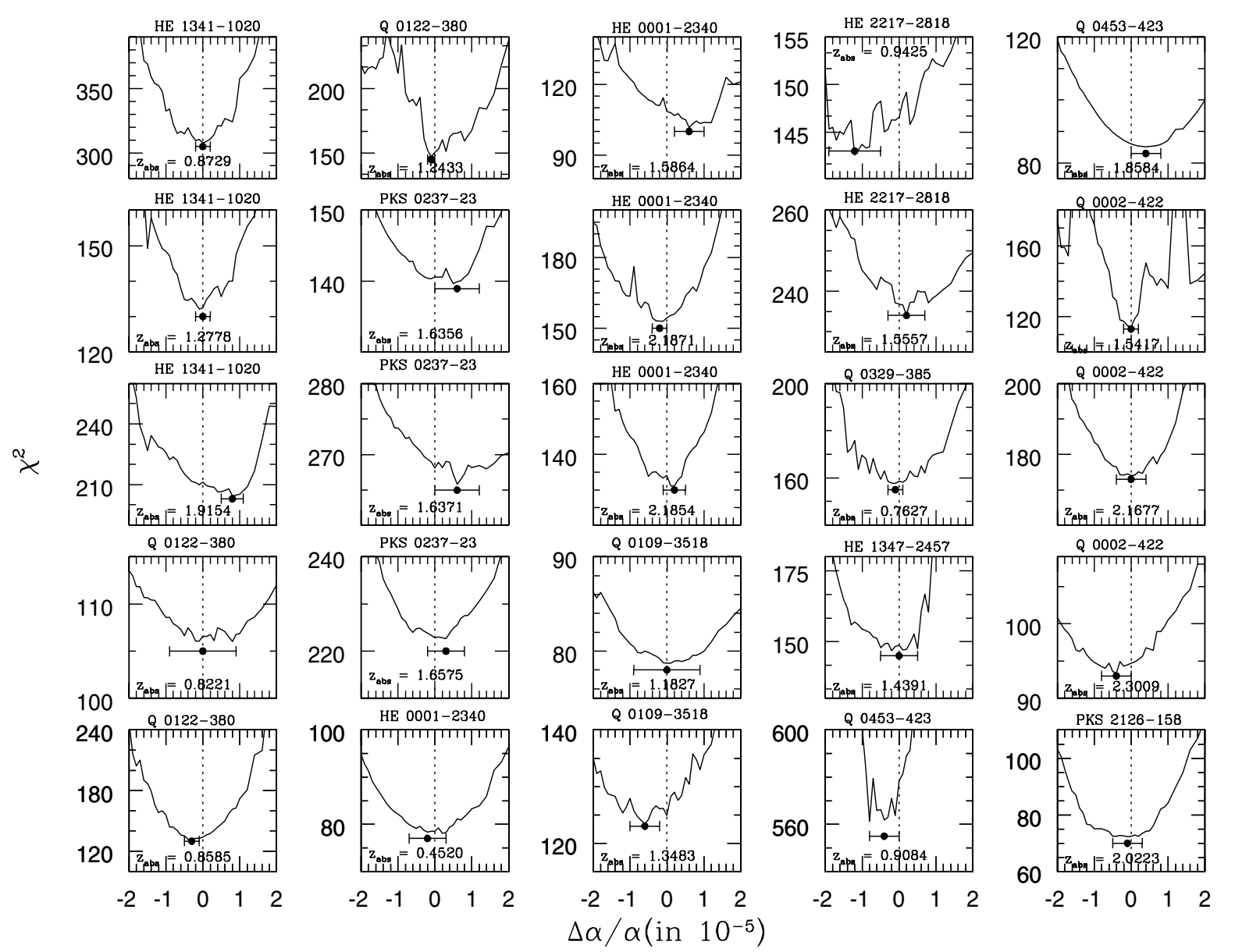

Fig. 14. Results from the UVES sample: $\chi^{2}$ is plotted as a function of $\Delta \alpha / \alpha$ for each system in our sample. The minima of the curves (marked with a dot) give the best fitted value $\Delta \alpha / \alpha$. The error in the measurement (error bar around the dot) is derived using $\Delta \chi^{2}=1$. Name of the QSOs and absorption redshifts $z_{\text {abs }}$ are given in each panel. The vertical dotted line in each panel marks the location of $\Delta \alpha / \alpha=0$. It is interesting to note that most of the systems are consistent with $\Delta \alpha / \alpha=0$ within the errors. Two of the systems $z_{\text {abs }}=1.5864$ toward HE $0001-2340$ and $z_{\text {abs }}=$ 0.7627 toward Q $0329-385$ are not considered in the analysis, as the only two components in these systems are blended as per our definition.

Mg II doublets and other anchors. Based on these preliminary fits a first set of parameters is generated to start the Voigt profile fitting procedure that includes $\Delta \alpha / \alpha$ variations as described in Sect. 3.3. In all our fits, we have constrained the redshifts parameter of all the species under consideration to be same. We do not know a priori whether the $b$ parameters of different species are identical or not. To take this uncertainty into account we perform the fit of the systems for two cases: (i) assuming that $b$ parameters of individual components are same for all species and (ii) assuming different $b$ parameters for different species. We determine the best-fit $\Delta \alpha / \alpha$ values for both cases. It is found that in all cases the two values are consistent with one another within $1 \sigma$ uncertainty. For our final result we consider the fit that gives the smaller reduced $\chi^{2}$.

\subsection{Determination of $\Delta \alpha / \alpha$}

The $\chi^{2}$ analysis for individual systems is shown in Fig. 14 . Here, we plot $\chi^{2}$ as a function of $\Delta \alpha / \alpha$. The dot with error-bar in each panel gives our best-fit estimate of $\Delta \alpha / \alpha$. The vertical line in each panel shows the value $\Delta \alpha / \alpha=0$. It is clear from the figure that apart from few cases the curve is well behaved and the best-fit values are consistent with zero in most cases. The numerical values of the fits are given in Table 3. In this table we list in the first four columns the name of the QSO, the emission redshift $\left(z_{\mathrm{em}}\right)$, absorption redshifts $\left(z_{\mathrm{abs}}\right)$ and velocity dispersions $b$ of individual components. When the best-fit solutions are obtained with different $b$ parameters the $b$ values quoted are for the main anchor line (Mg II, Si II or Al II lines). Column 5 lists the transitions used in the analysis with the same notations as in Table 1. $N_{\mathrm{c}}$ given in Col. 6 is the number of subcomponents used to fit the Fe II absorption profiles. The last two columns give the determination of $\Delta \alpha / \alpha$ obtained using laboratory wavelengths of $\mathrm{Mg}$ II, Mg I, and Si II absorption lines with terrestrial isotopic abundances (case 1) and with the wavelengths of the dominant isotopes (case 2, see next section). Numbers in brackets are the reduced $\chi^{2}$ for the fit. The numbers marked with an asterisk $\left(^{*}\right)$ are values obtained using different $b$ values for different species.

In Fig. 15 we plot individual determinations of $\Delta \alpha / \alpha$ as a function of $z_{\text {abs }}$. We also plot the existing results from the literature. The horizontal dotted line gives the weighted mean 
Table 3. Recovered value of $\Delta \alpha / \alpha$ in individual systems.

\begin{tabular}{|c|c|c|c|c|c|c|c|}
\hline \multirow[t]{2}{*}{ Name } & $\overline{z_{\mathrm{em}}}$ & $\overline{z_{\text {abs }}}$ & $\bar{b}$ & $\begin{array}{c}\text { Transitions } \\
\end{array}$ & $\overline{N_{\mathrm{c}}}$ & $\overline{\Delta \Delta \alpha / \alpha}$ & $\overline{\left.f 10^{-5}\right)}$ \\
\hline & & & & & & case 1 & case 2 \\
\hline HE 1341-1020 & 2.135 & $0.872666 \pm 0.000046$ & $5.65 \pm 0.13$ & abdfgi & 3 & $+0.0 \pm 0.2(1.19)$ & $-0.6 \pm 0.4(1.20)$ \\
\hline & & $0.872841 \pm 0.000212$ & $5.22 \pm 0.28$ & abdfg & & & \\
\hline & & $0.872903 \pm 0.000195$ & $3.28 \pm 0.28$ & abdfg & & & \\
\hline & & $0.872755 \pm 0.000266$ & $2.49 \pm 0.90$ & $a b$ & & & \\
\hline & & $0.872422 \pm 0.000089$ & $2.59 \pm 0.30$ & $a b$ & & & \\
\hline & & $0.872477 \pm 0.000563$ & 2.68 & $a b$ & & & \\
\hline & & $0.872568 \pm 0.000354$ & $7.65 \pm 0.84$ & $a b$ & & & \\
\hline & & $0.873007 \pm 0.000206$ & 0.50 & $a b$ & & & \\
\hline & & $1.277834 \pm 0.000584$ & $4.03 \pm 0.51$ & abdefh & 2 & $-0.1 \pm 0.2(1.01)$ & $+0.0 \pm 0.50(0.97)$ \\
\hline & & $1.277780 \pm 0.000571$ & $3.35 \pm 0.52$ & abdefh & & & \\
\hline & & $1.915216 \pm 0.000628$ & $2.57 \pm 0.73$ & cdefhkl & 4 & $+0.8 \pm 0.3(1.49)$ & $+0.6 \pm 0.3(1.48)$ \\
\hline & & $1.915330 \pm 0.000226$ & $3.40 \pm 0.23$ & cdefhkl & & & \\
\hline & & $1.915463 \pm 0.000302$ & $6.24 \pm 0.28$ & cdefhkl & & & \\
\hline & & $1.915561 \pm 0.002269$ & $3.86 \pm 1.44$ & cdefhkl & & & \\
\hline Q $0122-380$ & 2.190 & $0.822076 \pm 0.000032$ & $5.69 \pm 0.07$ & abdfghi & 1 & $+0.0 \pm 0.9(0.87)$ & $+0.0 \pm 0.9(0.87)$ \\
\hline & & $0.822182 \pm 0.000457$ & $6.41 \pm 1.28$ & $a b$ & & & \\
\hline & & $0.859270 \pm 0.000211$ & $3.92 \pm 0.08$ & abfghi & 2 & $-0.3 \pm 0.2(1.29)$ & $-0.5 \pm 0.3(1.18)$ \\
\hline & & $0.859318 \pm 0.000646$ & $3.42 \pm 0.13$ & abfghi & & & \\
\hline & & $1.243293 \pm 0.000109$ & $5.09 \pm 0.12$ & abdef & 3 & $-0.1 \pm 0.1(0.89)$ & $-0.5 \pm 0.1(1.01)$ \\
\hline & & $1.243374 \pm 0.000344$ & $3.12 \pm 0.50$ & abdef & & & \\
\hline & & $1.243483 \pm 0.000122$ & $6.83 \pm 0.41$ & abdef & & & \\
\hline & & $1.243589 \pm 0.000731$ & $5.20 \pm 1.03$ & $\mathrm{ab}$ & & & \\
\hline PKS 0237-23 & 2.222 & $1.635674 \pm 0.000708$ & $9.23 \pm 1.06$ & $a b k$ & 2 & $+0.2 \pm 0.7(0.82)^{*}$ & $-0.2 \pm 0.3(0.86)^{*}$ \\
\hline & & $1.635790 \pm 0.000132$ & $2.56 \pm 0.34$ & abdghk & & & \\
\hline & & $1.635883 \pm 0.000050$ & $4.04 \pm 0.80$ & abdghk & & & \\
\hline & & $1.636041 \pm 0.000152$ & $4.20 \pm 0.33$ & $\mathrm{abk}$ & & & \\
\hline & & $1.637128 \pm 0.000114$ & $3.60 \pm 0.42$ & abcdgh & 5 & $+0.6 \pm 0.6(1.16)^{*}$ & $-0.4 \pm 1.3(1.19)^{*}$ \\
\hline & & $1.636903 \pm 0.000119$ & $0.40 \pm 0.13$ & abdgh & & & \\
\hline & & $1.637331 \pm 0.000244$ & $3.87 \pm 0.62$ & abdgh & & & \\
\hline & & $1.637155 \pm 0.000209$ & $15.4 \pm 0.41$ & abcdgh & & & \\
\hline & & $1.637437 \pm 0.000170$ & $6.17 \pm 0.24$ & abdgh & & & \\
\hline & & $1.657390 \pm 0.000223$ & $5.46 \pm 0.34$ & dfjk & 8 & $+0.3 \pm 0.5(0.92)$ & $+0.3 \pm 0.7(0.92)$ \\
\hline & & $1.657172 \pm 0.000932$ & $5.51 \pm 0.92$ & $\mathrm{dfjk}$ & & & \\
\hline & & $1.657415 \pm 0.000348$ & $11.57 \pm 0.34$ & $\mathrm{dfjk}$ & & & \\
\hline & & $1.656864 \pm 0.001122$ & $10.42 \pm 1.22$ & $\mathrm{fjk}$ & & & \\
\hline & & $1.657066 \pm 0.001358$ & $4.75 \pm 1.34$ & fjk & & & \\
\hline & & $1.657723 \pm 0.000785$ & $6.91 \pm 1.02$ & $\mathrm{fjk}$ & & & \\
\hline & & $1.657884 \pm 0.000262$ & $5.36 \pm 0.28$ & dfjk & & & \\
\hline & & $1.657591 \pm 0.000622$ & $2.16 \pm 0.17$ & $\mathrm{dfjk}$ & & & \\
\hline HE 0001-2340 & 2.263 & $0.452060 \pm 0.000024$ & $1.74 \pm 0.05$ & abefhim & 1 & $+0.2 \pm 0.5(1.10)$ & $-0.3 \pm 0.5(1.21)$ \\
\hline & & $2.187149 \pm 0.001503$ & $2.03 \pm 0.23$ & bdegjk & 3 & $-0.2 \pm 0.2(1.20)$ & $-0.2 \pm 0.3(1.31)$ \\
\hline & & $2.187237 \pm 0.000129$ & $4.73 \pm 1.06$ & bdegjk & & & \\
\hline & & $2.187106 \pm 0.001420$ & $6.61 \pm 0.56$ & bdegjk & & & \\
\hline & & $2.185294 \pm 0.000089$ & $2.29 \pm 0.19$ & abdefjk & 2 & $+0.2 \pm 0.3(1.15)$ & $-0.2 \pm 0.3(1.17)$ \\
\hline & & $2.185452 \pm 0.000264$ & $3.48 \pm 0.52$ & abdefjk & & & \\
\hline & & $2.185256 \pm 0.000512$ & 1.51 & abjk & & & \\
\hline & & $2.185332 \pm 0.000839$ & 1.77 & abjk & & & \\
\hline Q 0109-3518 & 2.404 & $1.182683 \pm 0.000039$ & $3.04 \pm 0.09$ & abdfhi & 1 & $+0.0 \pm 0.8(0.98)$ & $-0.5 \pm 0.5(0.98)$ \\
\hline & & $1.182770 \pm 0.000389$ & $3.17 \pm 1.18$ & $\mathrm{ab}$ & & & \\
\hline & & $1.348308 \pm 0.000204$ & $2.28 \pm 0.21$ & defghijk & 2 & $-0.6 \pm 0.4(1.08)$ & $-0.6 \pm 0.4(1.08)$ \\
\hline & & $1.348346 \pm 0.000415$ & 3.72 & defghijk & & & \\
\hline HE 2217-2818 & 2.414 & $0.942330 \pm 0.000058$ & $4.91 \pm 0.12$ & abfh & 5 & $-1.2 \pm 0.7(0.90)^{*}$ & $-1.6 \pm 0.8(1.36)$ \\
\hline & & $0.942574 \pm 0.000067$ & $4.79 \pm 0.15$ & abfh & & & \\
\hline & & $0.942655 \pm 0.000119$ & $3.27 \pm 0.75$ & abfh & & & \\
\hline & & $0.942701 \pm 0.002092$ & $8.88 \pm 2.20$ & abfh & & & \\
\hline & & $0.942435 \pm 0.000454$ & $7.38 \pm 0.64$ & abfh & & & \\
\hline & & $0.942476 \pm 0.000282$ & 4.82 & abfh & & & \\
\hline & & $1.555425 \pm 0.000029$ & $2.52 \pm 0.06$ & $a b$ & 1 & $+0.2 \pm 0.5(1.22)^{*}$ & $+0.0 \pm 0.3(1.35)^{*}$ \\
\hline & & $1.555580 \pm 0.000098$ & $2.35 \pm 0.06$ & $a b$ & & & \\
\hline & & $1.555721 \pm 0.000178$ & $3.08 \pm 0.39$ & $a b$ & & & \\
\hline & & $1.555886 \pm 0.000041$ & $4.98 \pm 0.07$ & abdfghk & & & \\
\hline HE $1347-2457$ & 2.611 & 1.439541 & 40.91 & $\mathrm{k}$ & 3 & $-0.0 \pm 0.5(1.10)$ & $-0.4 \pm 0.5(1.11)$ \\
\hline & & $1.439219 \pm 0.002721$ & $14.2 \pm 0.90$ & dgkl & & & \\
\hline & & $1.439243 \pm 0.000365$ & $6.96 \pm 0.36$ & dgkl & & & \\
\hline & & $1.439349 \pm 0.000195$ & $2.61 \pm 0.07$ & dgkl & & & \\
\hline & & 1.438934 & 5.97 & $\mathrm{k}$ & & & \\
\hline Q $0453-423$ & 2.658 & $0.908131 \pm 0.000028$ & $2.91 \pm 0.10$ & abdfh & 8 & $-0.4 \pm 0.4(1.82)$ & $-0.4 \pm 0.3(1.93)$ \\
\hline & & $0.908225 \pm 0.000306$ & $3.62 \pm 0.76$ & abdfh & & & \\
\hline & & $0.908402 \pm 0.000044$ & $3.90 \pm 0.10$ & abdfh & & & \\
\hline & & $0.908490 \pm 0.000122$ & $7.43 \pm 0.33$ & abdfh & & & \\
\hline & & $0.908588 \pm 0.000069$ & $2.78 \pm 0.15$ & abdfh & & & \\
\hline & & $0.908634 \pm 0.000107$ & $16.31 \pm 0.22$ & abdfh & & & \\
\hline & & $0.908771 \pm 0.000122$ & $1.42 \pm 0.32$ & abdfh & & & \\
\hline & & 0.908266 & $22.97 \pm 2.58$ & $\mathrm{ab}$ & & & \\
\hline & & $1.858364 \pm 0.000078$ & $6.13 \pm 0.11$ & abdfh & 1 & $+0.4 \pm 0.4(1.13)$ & $+0.2 \pm 0.5(1.16)$ \\
\hline Q 0002-422 & 2.767 & $1.541474 \pm 0.000194$ & $7.61 \pm 0.34$ & $\mathrm{ab}$ & 3 & $+0.0 \pm 0.2(0.66)$ & $-0.3 \pm 0.2(0.65)$ \\
\hline & & $1.541799 \pm 0.000687$ & $11.86 \pm 0.70$ & abdfgh & & & \\
\hline & & $1.541869 \pm 0.000100$ & $4.09 \pm 0.18$ & abdfgh & & & \\
\hline & & $1.541935 \pm 0.000267$ & $5.28 \pm 0.24$ & abdfgh & & & \\
\hline & & $1.541605 \pm 0.000397$ & $4.55 \pm 0.91$ & $a b$ & & & \\
\hline & & $2.167849 \pm 0.000336$ & $3.42 \pm 0.28$ & cdfghjk & 2 & $+0.0 \pm 0.4(1.03)^{*}$ & $-0.7 \pm 0.4(1.03)^{*}$ \\
\hline & & $2.167783 \pm 0.000529$ & 2.568 & cdfghjk & & & \\
\hline & & $2.300832 \pm 0.000231$ & $4.78 \pm 0.19$ & cdjk & 3 & $-0.4 \pm 0.4(0.99)$ & $-0.4 \pm 0.4(1.13)$ \\
\hline & & $2.300997 \pm 0.000198$ & $5.61 \pm 0.15$ & $\mathrm{dfjk}$ & & & \\
\hline & & $2.300619 \pm 0.000111$ & $2.94 \pm 0.11$ & $\mathrm{dfjk}$ & & & \\
\hline PKS 2126-158 & 3.280 & $2.021923 \pm 0.000075$ & $4.87 \pm 0.10$ & bdfgk & 1 & $-0.1 \pm 0.4(1.19)$ & $-0.9 \pm 0.5(1.21)$ \\
\hline & & $2.022001 \pm 0.000612$ & 2.54 & $\mathrm{bk}$ & & & \\
\hline
\end{tabular}




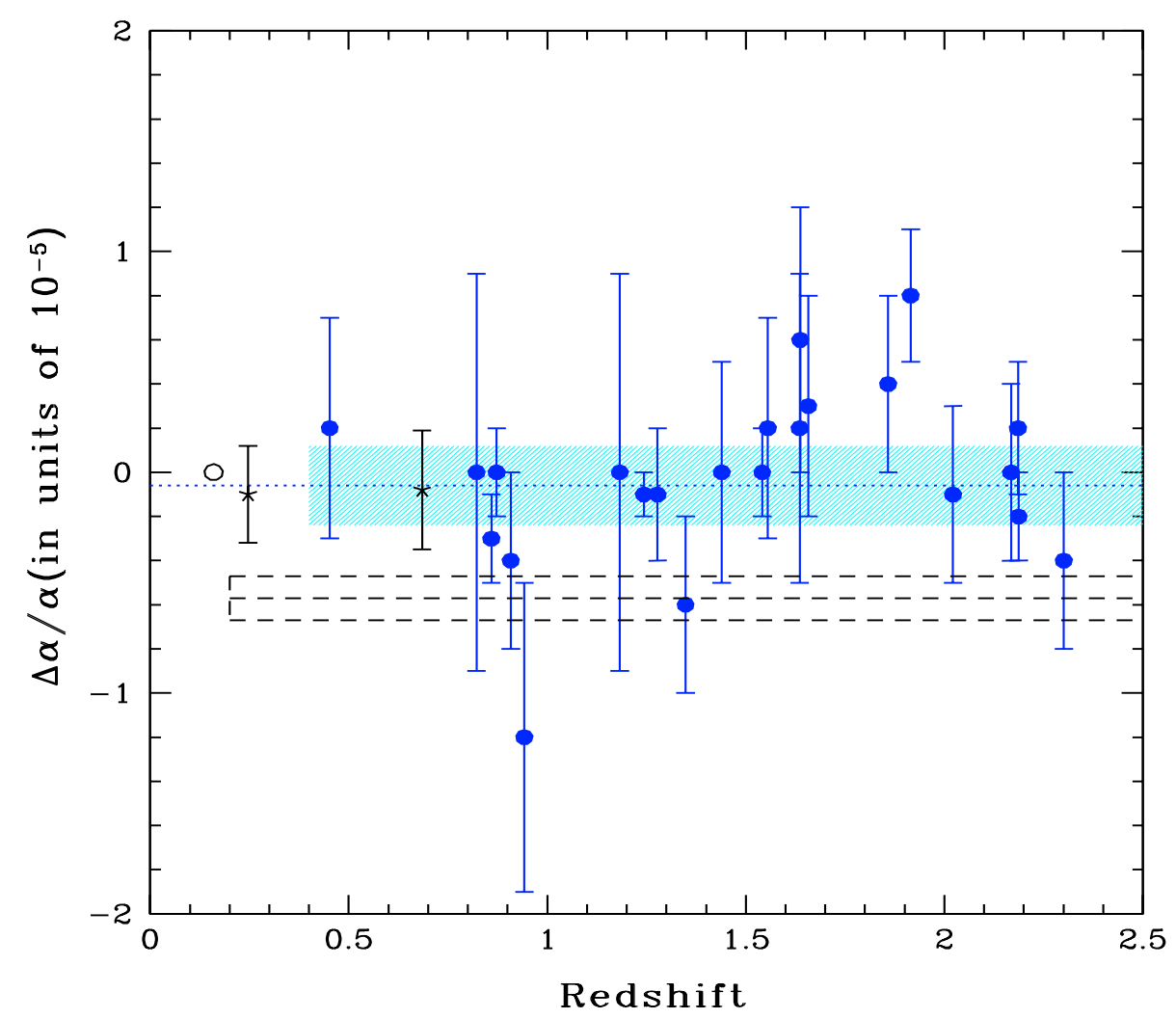

Fig. 15. Results from the UVES sample: the measured values of $\Delta \alpha / \alpha$ from our sample (filled circles) are plotted against the absorption redshifts of Mg II systems. Each point is the best-fit value obtained for individual systems using $\chi^{2}$ minimization as shown in Fig. 14. The open circle and stars are the measurements from the Oklo phenomenon and from molecular lines respectively. The weighted mean and $1 \sigma$ range measured by Murphy et al. (2003) are shown by the horizontal long dashed lines. Clearly most of our measurements are inconsistent with this range. The shaded region marks the weighted mean and its $3 \sigma$ error obtained from our study $\left[\langle\Delta \alpha / \alpha\rangle_{\mathrm{w}}=(-0.06 \pm 0.06) \times 10^{-5}\right]$. Our data give a $3 \sigma$ constraint on the variation of $\Delta \alpha / \alpha$ to be $-2.5 \times 10^{-16} \mathrm{yr}^{-1} \leq(\Delta \alpha / \alpha \Delta t) \leq 1.2 \times 10^{-16} \mathrm{yr}^{-1}$ in the case of a flat universe with $\Omega_{\lambda}=0.7, \Omega_{m}=$ 0.3 and $H_{0}=68 \mathrm{~km} \mathrm{~s}^{-1} \mathrm{Mpc}^{-1}$ for a median redshift of 1.55 .

of our sample with $1 /$ error $^{2}$ weighting. We estimate the error in the weighted mean using the standard equation,

Error in $x_{\mathrm{w}}=\sqrt{\frac{\sum_{\mathrm{i}}^{\mathrm{N}} w_{i}\left(x_{i}-x_{\mathrm{w}}\right)^{2}}{(N-1) \sum_{i}^{N} w_{i}}}$.

Here, $x_{\mathrm{w}}$ is the weighted mean of the variable $x_{i}$ of weight $w_{i}$ and $N$ refer to the size of sample. The shaded region passing through most of the error bars is our measured weighted mean and its $3 \sigma$ error. The weighted mean with $1 / \sigma^{2}$ weighting obtained for our sample is $(-0.06 \pm 0.06) \times 10^{-5}$ and the standard deviation in our measurements around the mean is $0.41 \times 10^{-5}$. All the points used are consistent with this weighted mean value with a reduced $\chi^{2}$ of 0.95 . As can be seen from the figure there are two points that deviate by more than $1 \sigma$ from the derived weighted mean value. These are from the $z_{\mathrm{abs}}=0.9425$ system toward HE 2217-2818 and the $z_{\mathrm{abs}}=$ 1.9154 system toward HE 1341-1020 (see also the $\chi^{2}$ curve for these systems in Fig. 14). As can be seen from Fig. 11, the $\mathrm{Fe}$ II lines in the $z_{\mathrm{abs}}=0.9425$ system are weak (barely above the cutoff). The reduced $\chi^{2}$ is dominated by uncertainties in fitting the $\mathrm{Mg}$ II profiles. In the case of the latter system the effect could just be due to the odd pixel in the Si II $\lambda 1808$ close to $v=$ $0 \mathrm{~km} \mathrm{~s}^{-1}$ (see Fig. 7).
Just for completeness we also fitted the weak systems that show at least two Fe II lines. The recovered values of $\Delta \alpha / \alpha$ based on these are given in Table 4 . As expected the individual measurements have large errors. The weighted mean value of $\Delta \alpha / \alpha$ measured from these systems is $-0.40 \pm 0.36$. Most of the individual measurements and the weighted mean are consistent with the value obtained from our main sample.

A summary of the results for different sub-samples is given in Table 5. The sample identification is given in the first column. The number of systems used and the median redshift of the samples are given in Cols. 2 and 3. The mean, weighted mean and $\sigma$ of the measured $\Delta \alpha / \alpha$ values are given in Cols. 4 , 5 , and 6 respectively. Last column gives the reduced $\chi_{\mathrm{W}}^{2}$ obtained from all our measurements for the measured weighted mean. It can be seen that there is no sub-sample in which we find a significant change in $\alpha$.

\subsection{The magnesium isotopic abundance}

One major uncertainty in the many-multiplet analysis comes from the determination of the effective rest wavelengths. Even though laboratory wavelengths are measured with a precision of a few $0.1 \mathrm{~m} \AA$, values given in Table 1 assume terrestrial 
Table 4. Results for the weak systems that show at least two detectable Fe II lines.

\begin{tabular}{llc}
\hline \hline \multicolumn{1}{c}{ QSO } & $z_{\text {abs }}$ & $\Delta \alpha / \alpha\left(\right.$ in $\left.10^{-5}\right)$ \\
\hline Q 0122-380 & 1.9102 & $-0.9 \pm 1.6$ \\
PKS 1448-232 & 1.5847 & $+0.4 \pm 2.0$ \\
PKS 0237-23 & 1.1846 & $-0.9 \pm 2.7$ \\
HE 0001-234 & 0.9489 & $-1.1 \pm 2.3$ \\
HE 2217-2818 & 1.6277 & $+0.3 \pm 0.6$ \\
Q 0329-385 & 1.4379 & $-0.4 \pm 0.6$ \\
HE 1347-2457 & 1.5082 & $-3.9 \pm 1.4$ \\
PKS 0329-255 & 0.9926 & $-0.3 \pm 1.3$ \\
HE 2347-4342 & 1.7962 & $-0.4 \pm 2.0$ \\
\hline
\end{tabular}

isotopic abundances. This assumption may not be valid at high redshift. In astrophysical settings the effect of isotopic shifts could be important for Si and Mg (see Murphy et al. 2003; Ashenfelter et al. 2003). Indeed, the relative abundances of different isotopes may depend on the overall metallicity of the gas. Gay \& Lambert (2000) have shown that the abundance of ${ }^{25} \mathrm{Mg}$ and ${ }^{26} \mathrm{Mg}$ relative to ${ }^{24} \mathrm{Mg}$ decreas with decreasing metallicity. In low-metallicity gas $\left(Z \leq 0.01 Z_{\odot}\right)$ most of the metals will be in their dominant isotopic state. Thus in the extreme case of very low metallicity, the effective rest wavelengths could take values in the range from terrestrial composite wavelength to wavelengths corresponding to the dominant isotope. This range is less than $0.5 \mathrm{~m} \AA$ for $\mathrm{Mg}$ I and $\mathrm{Si}$ II absorption lines but is of the order of $1 \mathrm{~m} \AA$ for $\mathrm{Mg}$ II lines. To accommodate this uncertainty we fit the systems using the wavelengths of the species from the dominant isotope. The measurements are given in the last column of Table 3. As expected, using these abundances leads to a lower $\alpha$ determination (see Table 5). Note however that even in this extreme case, the variation stays smaller than what has been claimed from previous studies. Note that the assumption of very low metallicity is extreme as: (i) the systems in the redshift range $0.4-2$ are more likely to have metallicity larger than $0.1 Z_{\odot}$ (e.g. Ledoux et al. 2002); (ii) the measured mean ratios of $\mathrm{Mg}^{24}: \mathrm{Mg}^{25}: \mathrm{Mg}^{26}$ in a cool dwarf with metallicity $Z=-1.5$ to $-1.0 Z_{\odot}$ is $80: 10: 10$ (from Table 1 of Yong et al. 2003). This gives weighted mean wavelengths close to the terrestrial wavelengths; and (iii) We also notice that the minimum $\chi^{2}$ for the fit in most cases (apart from 4 cases) is lower when we use the laboratory wavelengths in our analysis. Therefore, although some additional uncertainty and scatter could come from the isotopic abundances being different from that of terrestrial ones, our result using the laboratory wavelengths is most probably robust.

\section{Conclusion}

We have applied the MM method to a homogeneous sample of $50 \mathrm{Mg}$ II systems observed along 18 QSO lines of sight observed with UVES at the VLT. Using extensive simulations we show that our Voigt profile fitting procedure works well for the recovery of the input value of $\Delta \alpha / \alpha$ for simple single component systems. We can recover the input $\Delta \alpha / \alpha$ values with an error of $\sigma=0.23 \times 10^{-5}$ in the case of individual components for data of quality comparable to the UVES data $(S / N$ 70). We show that the uncertainty is about twice as large for data of $S / N \sim 30$ as used by previous surveys. In addition at this $S / N$ ratio, the use of strongly blended systems leads to larger uncertainties. We show that weak lines should be excluded from the determination as their use can lead to a false determination of $\Delta \alpha / \alpha$.

We devise the selection criteria for our sample using extensive simulations. This leads to 23 systems excluding stongly blended systems and systems with weak absorption lines. Individual systems and fits to all absorption lines are discussed in detail. We also avoided the two sub-DLAs from the analysis. In one of the systems that is in common with Murphy et al. (2003) our results are consistent with the earlier measurement albeit with smaller error (see Sect. 5.3.1).

The weighted mean value of the variation in the finestructure constant obtained from our analysis over the redshift range $0.4 \leq z \leq 2.3$ is $\Delta \alpha / \alpha=(-0.06 \pm 0.06) \times 10^{-5}$. For the median redshift of our sample $(z=1.54)$ we obtain a $3 \sigma$ constraint on the variation of $\Delta \alpha / \alpha$ to be $-2.5 \times 10^{-16} \mathrm{yr}^{-1} \leq$ $(\Delta \alpha / \alpha \Delta t) \leq 1.2 \times 10^{-16} \mathrm{yr}^{-1}$ in the case of a flat universe with $\Omega_{\lambda}=0.7, \Omega_{m}=0.3$ and $H_{0}=68 \mathrm{~km} \mathrm{~s}^{-1} \mathrm{Mpc}^{-1}$. To our knowledge this is the strongest constraint from QSO absorption line studies to date.

We show the effect of varying the isotopic abundances. Only if we assume isotopic abundances for very low metallicity would $\alpha$ show some variation. This is not acceptable for (i) assuming that all systems in the redshift range 0.4-2 have extremely low metallicity is incorrect as metals are seen in these systems and metallicity is measured to be larger than $0.1 Z_{\odot}$ (e.g. Ledoux et al. 2002); (ii) at $Z \sim 0.1 Z_{\odot}$, which would be a more reasonable assumption, the composite wavelength we get will be close to the laboratory value we have used in our study (we use Yong et al. 2003 for isotopic abundances). Therefore, although some additional uncertainty and scatter could come from the isotopic abundances being different from terrestrial ones, our result using laboratory wavelengths is most probably robust.

In conclusion, our study does not support claims by previous authors of a statistically significant change in $\Delta \alpha / \alpha$ with cosmic time at $z>0.5$. Our result still does allow smaller variations in excess of what is found based on the Oklo phenomenon. Future very high resolution $(R \sim 100000)$ spectroscopic studies are needed to probe the variations in $\alpha$ with much better accuracy.

Acknowledgements. This work is based on observations collected during programme 166.A-0106 (PI: Jacqueline Bergeron) of the European Southern Observatory with the Ultra-violet and Visible Echelle Spectrograph mounted on the 8.2 m KUEYEN telescope operated at the Paranal Observatory, Chile. P.P.J. thanks E. VangioniFlam and J. P. Uzan for fruitful discussions. H.C. thanks CSIR, INDIA for the grant award No. 9/545(18)/2KI/EMR-I. R.S. thanks CNRS/IAP for the hospitality. We gratefully acknowledge support from the Indo-French Centre for the Promotion of Advanced Research (Centre Franco-Indien pour la Promotion de la Recherche Avancée) under contract No. 3004-A. 
Table 5. Summary of results for various sub-samples.

\begin{tabular}{lcccccc}
\hline \hline \multicolumn{1}{c}{ Sample } & $\begin{array}{c}\text { Number of } \\
\text { systems }\end{array}$ & $z$ & Mean & $\begin{array}{c}\Delta \alpha / \alpha\left(10^{-5}\right) \\
\text { weighted mean }\end{array}$ & \multicolumn{2}{c}{$\chi_{\mathrm{W}}^{2}$} \\
\hline Single + double (case 1) & 12 & 1.54 & $+0.01 \pm 0.15$ & $-0.08 \pm 0.07$ & 0.27 & 0.55 \\
Full sample (case 1) & 23 & 1.54 & $-0.02 \pm 0.10$ & $-0.06 \pm 0.06$ & 0.41 & 0.95 \\
Weak (case 1) & 9 & 1.51 & $-0.80 \pm 0.58$ & $-0.40 \pm 0.36$ & 1.27 & 1.00 \\
Weak + All (case 1) & 32 & 1.51 & $-0.24 \pm 0.18$ & $-0.07 \pm 0.06$ & 0.81 & 0.96 \\
Single + double (case 2) & 12 & 1.54 & $-0.31 \pm 0.14$ & $-0.33 \pm 0.09$ & 0.33 & 0.63 \\
Full Sample (case 2) & 23 & 1.54 & $-0.33 \pm 0.11$ & $-0.36 \pm 0.06$ & 0.44 & 1.06 \\
\hline
\end{tabular}

Case 1: laboratory wavelengths given in Table 1 are used.

Case 2: rest wavelengths of dominant isotopes for $\mathrm{Mg}$ and $\mathrm{Si}$ are used.

\section{References}

Ashenfelter, T., Mathews, G. J., \& Olive, K. A. 2003 [astro-ph/0309197]

Bahcall, J. N., Sargent, W. L. W., \& Schmidt, M. 1967, ApJ, 149, L11

Bahcall, J. N., Steinhardt, C. L., \& Schlegel, D. 2004, ApJ, 600, 520

Bergeson, S. D., Mullman, K. L., Wickliffe, W. E., et al. 1996, ApJ, 464, 1044

Chengalur, J. N., \& Kanekar, N. 2003 [astro-ph/0310764]

Cowie, L. L., \& Songaila, A. 1995, ApJ, 453, 596

Dzuba, V. A., Flambaum, V. V., Kozlov, M. G., \& Marchenko, M. 2002, Phys. Rev. A, 66, 022501

Dzuba, V. A., Flambaum, V. V., \& Webb, J. K. 1999, PRL, 82, 888

Edlén, B. 1966, Metrologia, 2, 71

Gay, P., \& Lambert, F. L. 2000, ApJ, 533, 260

Griesmann, U., \& Kling, R. 2000, ApJ, 536, L113

Khare, P., Srianand, R., York, D. G., et al. 1997, MNRAS, 285, 167

Ledoux, C., Bergeron, J., \& Petitjean, P. 2002, A\&A, 385, 802

Levshakov, S. A. 1994, MNRAS, 269, 339

Mohr, P. J., \& Taylor, B. N. 2000, Rev. Mod. Phys., 72, 351

Morton, D. C. 1991, ApJS, 77, 119

Morton, D. C. 1992, ApJS, 81, 883

Murphy, M. T., Webb, J., Flambaum, V., Prochaska, J. X., \& Wolfe, A. M. 2001, MNRAS, 327, 1237

Murphy, M. T., Webb, J., Flambaum, V., et al. 2001a, MNRAS, 327, 1244
Murphy, M. T., Webb, J. K., \& Flambaum, V. V. 2003, MNRAS, 345, 609

Nave, G., Learner, R. C. M., Thorne, A. P., et al. 1991, J. Opt. Soc. Am. B, 8, 2028

Petitjean, P., \& Aracil, B. 2003, A\&A, submitted

Pickering, J. C., Thorne, A. P., Murray, J. E., et al. 2002, MNRAS, 319,163

Pickering, J. C., Thorne, A. P., \& Webb, J. K. 1998, MNRAS, 300, 131

Potekhin, A. Y., \& Varshalovich, D. A. 1994, A\&AS, 104, 89

Press, W., Teukolsky, S. A., Vellerling, W. T., et al. 2000, Numerical Recipes in Fortran: The art of Scientific Computing (Foundation Books), 690

Prochaska, J. X., Wolfe, A. M., Tytler, D., et al. 2001, ApJS, 137, 21

Srianand, R., \& Khare, P. 1993, ApJ, 413, 486

Stumpff, P. 1980, A\&AS, 41, 1

Uzan, J. 2003, RvMP, 75, 403

Varshalovich, D. A., Panchuk, V. E., \& Ivanchik, A. V. 1996, Astron. Lett., 22, 6

Webb, J. K., Murphy, M. T., Flambaum, V. V., et al. 2001, PRL, 87, 091301

Wolfe, A. M., Brown, R. L., \& Roberts, M. S. 1976, Phys. Rev. Lett., 37,177

Yong, D., Grundahl, F., Lambert, D. L., Nissen, P. E., \& Shetrone, M. D. 2003, A\&A, 402, 985 